\title{
Comprehensive development, uncertainty and sensitivity analysis of a model for the
} hydrolysis of rapeseed oil

Forero-Hernandez, Hector; Jones, Mark Nicholas; Sarup, Bent; Jensen, Anker Degn; Abildskov, Jens; Sin, Gürkan

\section{Published in:}

Computers \& Chemical Engineering

Link to article, DOI:

10.1016/j.compchemeng.2019.106631

Publication date:

2020

Document Version

Peer reviewed version

Link back to DTU Orbit

Citation (APA):

Forero-Hernandez, H., Jones, M. N., Sarup, B., Jensen, A. D., Abildskov, J., \& Sin, G. (2020). Comprehensive development, uncertainty and sensitivity analysis of a model for the hydrolysis of rapeseed oil. Computers \& Chemical Engineering, 133, [106631]. https://doi.org/10.1016/j.compchemeng.2019.106631

\section{General rights}

Copyright and moral rights for the publications made accessible in the public portal are retained by the authors and/or other copyright owners and it is a condition of accessing publications that users recognise and abide by the legal requirements associated with these rights.

- Users may download and print one copy of any publication from the public portal for the purpose of private study or research.

- You may not further distribute the material or use it for any profit-making activity or commercial gain

- You may freely distribute the URL identifying the publication in the public portal 


\title{
Comprehensive Development, Uncertainty and Sensitivity Analysis of a Model for the Hydrolysis of Rapeseed Oil
}

\author{
Hector Forero-Hernandez ${ }^{\mathrm{a}, \mathrm{b}}$, Mark Nicholas Jones ${ }^{\mathrm{a}, \mathrm{b}}$, Bent Sarup ${ }^{\mathrm{a}}$, Anker \\ Degn Jensen ${ }^{\mathrm{c}}$, Jens Abildskov ${ }^{\mathrm{b}}$, Gürkan Sin ${ }^{\mathrm{b}, *}$ \\ ${ }^{a}$ Edible Oil Systems, Alfa Laval Copenhagen A/S, 2860 Søborg, Denmark \\ ${ }^{b}$ Process and Systems Engineering Research Centre (PROSYS), Department of Chemical \\ and Biochemical Engineering, Technical University of Denmark, 2800 Kongens Lyngby, \\ Denmark \\ ${ }^{c}$ Combustion and Harmful Emission Control Research Centre (CHEC), Department of \\ Chemical and Biochemical Engineering, Technical University of Denmark, 2800 Kongens \\ Lyngby, Denmark
}

\begin{abstract}
A model describing the batch hydrolysis of rapeseed oil including kinetics and mass transfer at subcritical conditions is presented in this paper. The primary purpose of this model is to interpret experimental data collected from typical batch tests and to estimate model parameters. The developed model was further investigated using Monte Carlo simulations to statistically quantify the variability in the model outputs due to uncertainties in the parameter estimates. To understand which parameters in the model are responsible for the output uncertainty, a sensitivity analysis method was used (polynomial chaos expansionsbased Sobol sensitivity indices). The results from the sensitivity analysis helped to identify what parameters in the model are influential, giving insight into the robustness and predictive capabilities of the model which form the basis for any model-based decision making for detailed process characterization, design, optimization and operation of the hydrolysis of rapeseed oil.
\end{abstract}

Keywords: Uncertainty Analysis, Sensitivity Analysis, Vegetable Oils, Kinetic modeling

\footnotetext{
${ }^{*}$ Corresponding author

Email address: gsi@kt.dtu.dk (Gürkan Sin)
} 


\section{Introduction}

While production of oilseed and vegetable oils, with an estimated production of 769 million metric tons per year (2017/2018 forecast), are small compared to primary petrochemicals, they are an important component in today's commodi5 ties market [1]. To a large extent, this is due to the high technological standard of this mature industry [2]. Generally speaking, vegetable oils are extracted from soybeans, palm fruit, sunflower, coconuts, rapeseed, cottonseed, olives, flax, castor seed and groundnuts, making them the most important renewable raw materials for the chemical industry [3].

10

Vegetable oils are primarily triglycerides, i.e., triesters of long-chain saturated and unsaturated fatty acids with glycerol. Basic oleochemicals (chemicals derived from vegetable oil and animal fats) are free fatty acids, methyl esters, fatty alcohols, and fatty amides as well as glycerol as by product [4]. Fatty acids of different chain length, saturated and unsaturated, have been produced through hydrolysis on an industrial scale for more than 130 years (Lemmens Fryer's Process, Budde and Robertson's Process, Ittner's Process, Twitchell Process, etc.)[5]. The hydrolysis of fats and oils also produces several important industrial chemicals including monoglycerides, diglycerides, and glycerol as side-products [6]. The existing industrial and commercial process hydrolyzes oils to fatty acids and glycerol at temperature and pressure of about $250^{\circ} \mathrm{C}$ and 50 bar within a few hours to achieve conversions between $96 \%$ and $99 \%$ [7, 8].

The main products of the oleochemical industry are for human consumption (ca. 80\%) and nonfood applications (ca. 20\%) such as detergents, cosmetics, plastics, or biofuels. Because of their importance in the preparations of further derivatives and with a fast-growing market for bio-based products, demand for these products is expected to grow around the world [9]. For example, the global market for natural fatty acids reached US $\$ 17.1$ billion and US $\$ 18.3$ billion in 2014 and 2015, respectively. This market is expected to grow at an annual growth rate of $7.1 \%$ to US $\$ 25.7$ billion for the period 2014-2019 [10]. Hence, it has become more imperative for industry to better respond to consumer needs 
by understanding the chemical transformations involved in the processing of vegetable oils.

\subsection{Previous modeling efforts}

The hydrolysis of oils and fats is an important processing route for the chemthe hydrolysis reaction of triglycerides [11, 12, 13, 14, 15. The process for hydrolyzing oil by using water is influenced by both mass transfer between the phases and by the kinetics of the reactions. Processes at subcritical conditions are conducted with water in a temperature range of 100 to $374^{\circ} \mathrm{C}$ under suffiConventional industrial hydrolysis of oils and fats is generally conducted under subcritical pressures close to 45 bar and temperatures around $250^{\circ} \mathrm{C}$ for a maximum period of 2 hours with high yields (96-99\%). The resulting products are extremely dark fatty acids and an aqueous solution rich in glycerol, which 45 need to be re-distilled for color removal and purification [? ]. The hydrolysis of triglycerides can be carried out batchwise (Twitchell process) or continuously (Colgate Emery process) [16].

Currently, the continuous hydrolysis of triglycerides allows the industrial production of fatty acids by the action of water vapor in a spray column at high pressures [17]. The reaction is non-catalyzed with conversions of triglycerides up to $99 \%$ after 1 to 3 hours in the reactor [18. The glycerol formed during the hydrolysis is continuously extracted from the reaction medium.

[19, 20] found that the conversion of this reaction is dependent of the temperature. [6] described the autocatalytic behavior of the reaction in a batch reactor, with the assumption that the reaction occurs in the bulk of the oil phase. Such behavior had been attributed to the elevated ion product of water at high temperature [6. 13, 14, 15, proposed a model to describe the biphasic hydrolysis by using a combined mass transfer and kinetic approach. The authors also investigated the reaction in both batch and continuous mode and 6o proposed a three-step reversible reaction mechanism for the hydrolysis of tri-, 
di- and monoglycerides [13, 14, 15]. [12] developed a second-order chemical reaction model and suggested an autocatalytic mechanism due to the action of generated fatty acids in the aqueous phase that subsequently act as acid catalyst in subcritical water [12]. More recently, [21] presented a kinetic study for the 65 hydrolysis of sunflower oil under subcritical conditions in a PFR, where information on the different kinetic regimes the reaction exhibits as well as on the rate parameters was also provided. Additionally, the hydrolysis at low/middle temperatures over solid acid catalists was discussed by [22]. In their study, the rates of hydrolysis were increased by the action of solvents and phase transfer agents.

Nevertheless, after a revision of the technical process development of hydrolysis of triglycerides one finds that some basic facts related to the thermodynamics, mass transfer, and kinetics inherent to the biphasic nature of the process have only been partially recognized due to the rather harsh physical conditions at which tri-, di-, and monoglycerides are exposed to during their hydrolysis. In the literature, the experimental data available which concerns the reactions needed for the design and analysis of processes which involves lipid technology are, in the best case scenario, scarce and not conclusive such as, solution and reaction properties of the species involved, extent of miscibility, phases where so reactions occur and the reaction and mass transfer mechanisms. Moreover, the mathematical models used to correlate the behavior of reactions with phenomena are not accurate enough and are not used for validation. Hence, these models are not predictive in nature, which limit their scientific and industrial applicability. Hence, only limited information is available 23. Besides, another disadvantage of the available models in the open literature is, to the best of our knowledge, that none of the recent studies has been analyzed and thoroughly validated.

Kinetic modeling and validation of biphasic reactions, specifically the hydrolysis of vegetable oils at subcritical conditions, is complicated due to the so heterogeneous nature of the system and the lack of experimental information. Moreover, these task depends strongly upon selecting accurate estimation and 
simulation methods. Missing or insufficient physical properties and operating parameters can weaken the accuracy of a model or even prevent one from simulating it [24]. Hence, it is very important to find and use good values for models, parameters and properties. In this way, integrating experimentation and modeling of chemical processes is a relevant validation strategy for improving property and process modeling as well as designing and optimizing the reaction and recovery of high value added products.

In this work, a problem-specific model was proposed to evaluate its applicability and predictive capabilities. In the modeling of biphasic reaction systems, a model can be used for studying the behavior of the different species within one phase, defining the distribution of species between two phases, or predicting the effect of process conditions on reacting species or reaction rates. The challenges presented by the absence of accurate models for this reaction and process can be overcome by collecting data through experiments, rigorous mathematical modeling, uncertainty and sensitivity quantification, thermodynamic analysis of reactions and mixtures, simulation of technologies and scenarios, and analysis of fluid flow behavior in industrial settings. By applying these methodologies, it is possible to represent accurately a studied system. In this way, the production of fatty acids can be analyzed in terms of its phenomena, design variables and process parameters as a whole. Furthermore, the results can be used to validate and improve property and process modeling.

This model describes batch runs with a reasonable number of parameters and degree of accuracy. Monte Carlo simulations were run to identify the working bounds and the cases where the model is most accurate under uncertainty in the parameter estimates and model outputs. Furthermore, a sensitivity analysis method based on variance was used in order to quantify how much of the variance in the model output each uncertain parameter is responsible for. 


\section{Model development}

\subsection{System phenomena relevant for modeling}

The hydrolysis of triglycerides is generally considered a three-step set of consecutive, reversible reactions in which one mole of glyceride is generated and consumed as seen in Reaction 1) - Reaction 3). In water at high temperature, this hydrolysis reaction with its backward reaction occurs without any catalyst. In this system of reversible reactions, a molecule of Triglyceride $(T G)$ is hydrolyzed to one molecule of Diglyceride $(D G)$ and one molecule of Fatty Acid $(F A) . D G$ is subsequently hydrolyzed to Monoglyceride $(M G)$ which is further hydrolyzed to Glycerol (Gly), producing three molecules of $F A$ in total [14, 25].

The following reactions present the three-step vegetable oil hydrolysis reaction:

$$
\begin{array}{ll}
\mathrm{TG}+\mathrm{H}_{2} \mathrm{O} \underset{\mathrm{k}_{-1}}{\stackrel{\mathrm{k}_{1}}{\rightleftharpoons}} \mathrm{FA}+\mathrm{DG} & \text { (Reaction 1) } \\
\mathrm{DG}+\mathrm{H}_{2} \mathrm{O} \underset{\mathrm{k}_{-2}}{\stackrel{\mathrm{k}_{2}}{\rightleftharpoons}} \mathrm{FA}+\mathrm{MG} & \text { (Reaction 2) } \\
\mathrm{MG}+\mathrm{H}_{2} \mathrm{O} \underset{\mathrm{k}_{-3}}{\stackrel{\mathrm{k}_{3}}{\rightleftharpoons}} \mathrm{FA}+\mathrm{Gly} & \text { (Reaction 3) }
\end{array}
$$

130

However, as presented by Noureddini et al, the reaction described by (Reaction 4) can take place at high temperatures $\left(180^{\circ} \mathrm{C}-280^{\circ} \mathrm{C}\right)[26$.

$$
\mathrm{TG}+\mathrm{MG} \underset{\mathrm{k}_{-4}}{\stackrel{\mathrm{k}_{4}}{\rightleftharpoons}} 2 \mathrm{DG} \quad \text { (Reaction 4) }
$$

As a result of its heterogeneous nature, the hydrolysis reaction is affected not only by the chemical kinetics but also by the rate of mass transfer between the oil and water phase. Other important variables which are affecting the process are temperature, pressure, density, viscosity, and geometry of reactor. Since the polar water and non-polar $T G$ form two immiscible phases, one component 
must diffuse into the other before the reaction between them can happen. Thus, both a mass transfer of water from the bulk of the aqueous phase to the organic phase and a chemical reaction take place in the process. An increase in the solubility of water in the oil-rich phase with high temperature as well as the action of high agitation could overcome the interface mass-transfer resistance due to the dissimilarity in size and polarity between triglycerides and water, and consequently boost the reaction rate of hydrolysis. At subcritical conditions the dielectric constant of water declines significantly as water is heated at constant pressure. At such conditions, water behaves closely as organic solvent. This means that its solvation properties are enhanced because the hydrogen bonding between molecules of water is weaker allowing greater miscibility and 150 consequently diffusion of. Additionally, different levels of agitation can result in the change of dimension and nature of the interfacial area between organic and aqueous phase.

(19) observed that in the beginning of the reaction, a water in oil emulsion is formed and the reaction proceeds slowly due to mass transfer limitations. As the reaction continues, the emulsion breaks down and the reaction rate increases significantly. This is because the fatty acid content in the oil increases due to the reaction, which then acts as an acid catalyst. Consequently, the overall reaction rate expression should consist of the mass transfer rate, the chemical reaction rate and include explicitly the solubility of water in the oil phase depending on its composition.

According to the two-film theory, an interface separates the phases and there is one film in either phase that adheres to the interface [27, 28]. Mass transfer and reaction occur through the following consecutive steps as proposed by [29] for a high-pressure oil-hydrolysis countercurrent spray reactor:

1. Water in the aqueous bulk diffuses through the aqueous film.

2. Water diffuses through the liquid/liquid interface.

3. Water diffuses through the oil film to the oil phase bulk where the reaction takes place. 
4. Water reacts with the tri-, di-, and monoglycerides forming fatty acids

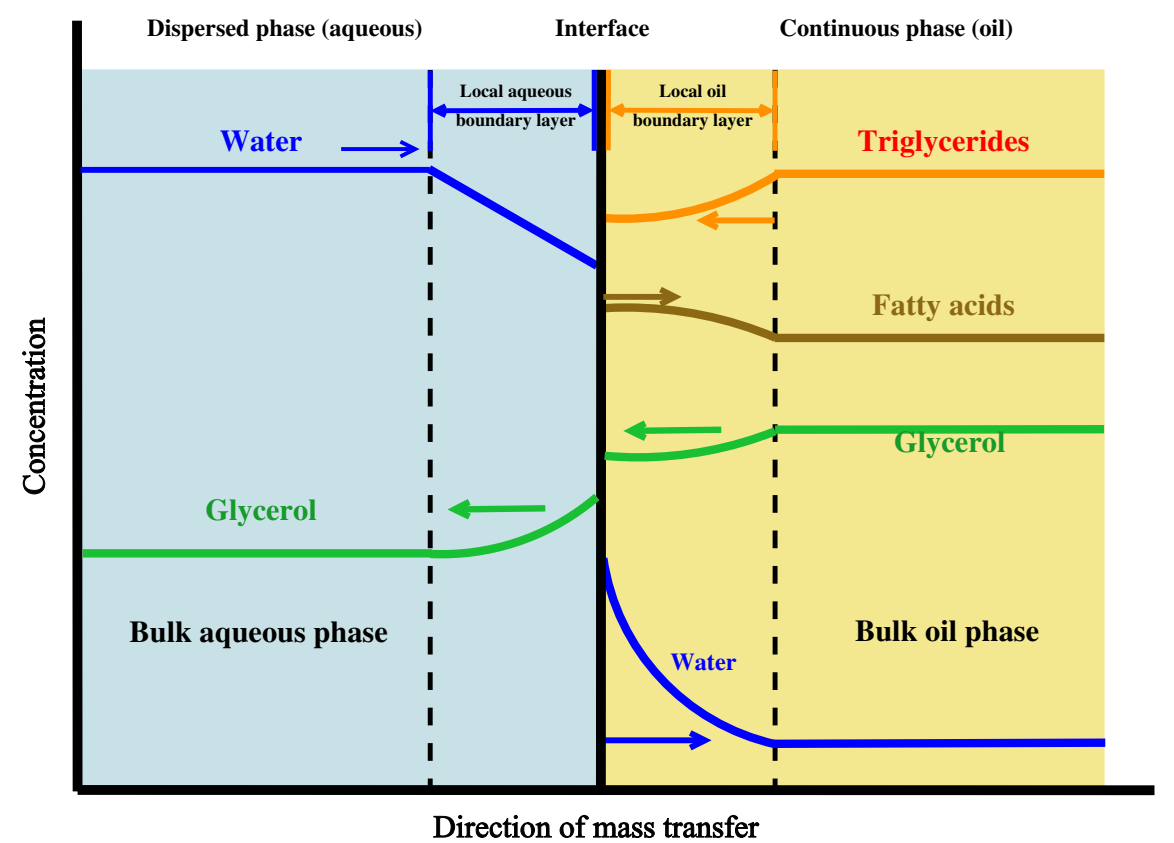

Figure 1: Mass transfer and reaction processes based on the two film theory for the hydrolysis of oils

\subsection{Mathematical formulation}

The general modeling objective followed in this work is that the model should be sophisticated enough to describe the complexities of the hydrolysis of vegetable oil, but the parameters should be based on measurable phenomena to a 
1. Two phases are present: aqueous (polar) and oil (non-polar) represented by the subscripts $a q$ and oil respectively. All the reactions occur in oil phase.

2. Reactions are elementary and consecutive.

3. As the reactions are reversible, $T G, D G$, and $M G$ are present at the chemical equilibrium.

4. Partition of water and glycerol among the two phases.

5. Mass transfer phenomena is described by Whitman's two-film theory [30].

Based on the reaction scheme presented in (Reaction 1) to (Reaction 4), the following kinetic equations are proposed:

$$
\begin{array}{r}
\mathrm{TG}+\mathrm{H}_{2} \mathrm{O} \underset{\mathrm{k}_{-1}}{\stackrel{\mathrm{k}_{1}}{\rightleftharpoons}} \mathrm{FA}+\mathrm{DG} \\
R_{1}=k_{1} \cdot C_{T G} \cdot C_{H_{2} O}-k_{-1} \cdot C_{D G} \cdot C_{F A} \\
\mathrm{DG}+\mathrm{H}_{2} \mathrm{O} \underset{\mathrm{k}_{-2}}{\stackrel{\mathrm{k}_{2}}{\rightleftharpoons}} \mathrm{FA}+\mathrm{MG} \\
R_{2}=k_{2} \cdot C_{D G} \cdot C_{H_{2} O}-k_{-2} \cdot C_{M G} \cdot C_{F A} \\
R_{3}=k_{3} \cdot C_{M G} \cdot C_{H_{2} O}-k_{-3} \cdot C_{G l y} \cdot C_{F A} \\
\mathrm{MG}+\mathrm{MG} \underset{\mathrm{k}_{-3}}{\stackrel{\mathrm{k}_{4}}{\rightleftharpoons}} 2 \mathrm{DG} \\
R_{4}=k_{4} \cdot C_{T G} \cdot C_{M G}-k_{-4} \cdot C_{D G}^{2}
\end{array}
$$

Where $k_{i}$ is the reaction rate constant for component $i$.

In order to model the batch hydrolysis, the expression for every species involved in the reaction should be determined. The reaction rates can be described by the expressions in Equations (1) - (4). The mole balances for the aqueous and oil phases become: 
- Aqueous phase

$$
\begin{gathered}
\frac{d n_{\mathrm{H}_{2} \mathrm{O}}^{a q}}{d t}=-J_{\mathrm{H}_{2} \mathrm{O}}^{a q} \cdot V_{a q} \\
\frac{d n_{G l y}^{a q}}{d t}=J_{G l y}^{a q} \cdot V_{a q}
\end{gathered}
$$

- Oil phase

$$
\begin{gathered}
\frac{d n_{T G}^{o i l}}{d t}=\left(-R_{1}-R_{4}\right) \cdot V_{\text {oil }} \\
\frac{d n_{D G}^{\text {oil }}}{d t}=\left(R_{1}-R_{2}-2 \cdot R_{3}\right) \cdot V_{\text {oil }} \\
\frac{d n_{M G}^{o i l}}{d t}=\left(R_{2}-R_{3}-R_{4}\right) \cdot V_{\text {oil }} \\
\frac{d n_{F A}^{o i l}}{d t}=\left(R_{1}+R_{2}+R_{3}\right) \cdot V_{\text {oil }} \\
\frac{d n_{H_{2} O}^{\text {oil }}}{d t}=\left(-R_{1}-R_{2}-R_{3}+J_{H_{2} O}^{\text {oil }}\right) \cdot V_{\text {oil }} \\
\frac{d n_{G l y}^{o i l}}{d t}=\left(R_{3}-J_{G l y}^{o i l}\right) \cdot V_{o i l}
\end{gathered}
$$

The equations which describe the mass transfer of water and glycerol from and to the two phases are as follows:

$$
\begin{gathered}
J_{\mathrm{H}_{2} \mathrm{O}}^{a q}=k_{1 L, a} \cdot\left(C_{\mathrm{H}_{2} \mathrm{O}}^{a q}-m_{\mathrm{H}_{2} \mathrm{O}} \cdot C_{\mathrm{H}_{2} \mathrm{O}}^{o i l, *}\right) \\
J_{G l y}^{a q}=k_{2 L, a} \cdot\left(C_{G l y}^{a q}-m_{G l y} \cdot C_{G l y}^{o i l, *}\right) \\
J_{H_{2} \mathrm{O}}^{o i l}=k_{1 L, a} \cdot\left(C_{\mathrm{H}_{2} \mathrm{O}}^{o i l, *} C_{H_{2} \mathrm{O}}^{o i l}\right) \\
J_{G l y}^{o i l}=k_{2 L, a} \cdot\left(C_{G l y}^{o i l}-C_{G l y}^{o i l, *}\right)
\end{gathered}
$$


When one assums steady-state, the interfacial concentration $\left(C_{i}^{*}\right)$ can be calculated by solving the following balance for the $i$ th component:

$$
J_{i}^{a q} \cdot V^{a q}=J_{i}^{o i l} \cdot V^{o i l}
$$

$$
V_{\text {oil }}=\sum_{i, o i l} \frac{n_{i, o i l}}{\rho_{i}} \quad V_{a q}=\sum_{i, a q} \frac{n_{i, a q}}{\rho_{i}}
$$

Densities of components $\left(\rho_{i}\right)$ are calculated by using polynomial fittings of experimental data of density versus temperature for the desired range. Corre-

In the absence of experimental data for the liquid-liquid equilibrium, Excess Gibbs energy-based activity coefficients are used to predict the partition coefficient $\mathrm{m}$. In this study the modified UNIFAC (Dortmund) model developed by Gmehling et al. is applied for the calculation of the equilibrium compositions of the two liquid phases and partition coefficients [31, 32, 33, 34, 35. This method was chosen due to its large range of applicability and the reliable results predicted for properties related to phase equilibria of systems involving vegetable oils [36]. Moreover, thermodynamic models such as NRTL, UNIQUAC, SAFT or CPA require specific binary interaction parameters obtained by regression and extrapolation of experimental phase equilibrium data of systems containing tri-, di- and monoglycerides which, unfortunately, are not available in the open literature. In order to solve the mass transfer equations, it is also necessary to define the partition coefficient for both water and glycerol. . These calculations are carried out by using the iterative algorithm proposed by [37, and it is

Volumes of oil and aqueous phase are calculated by using the following equation: lation parameters for these polynomials have been obtained from the CAPEC 
Lipids Database [38]. In brief, the mathematical model has 8 variables, i.e. moles, and it contains 10 parameters which are estimated from dedicated experiments.

\section{Experimental materials and methods}

\subsection{Batch experiments}

Hydrolysis reactions were run in a $300 \mathrm{ml}$ Hastelloy C-276 jacketed reactor with ceramic band heaters (Parker Autoclave Engineers, Model $300 \mathrm{ml}$ HC EZE-Seal). The reactor includes a spiral cooling coil to provide a mean of removing heat from the vessel to control the reaction and for cooling the reactor at the end of every experiment. This coil consists of multiple loops wound in the inside diameter of the vessel around the shaft guide. The autoclave was sealed and flushed 3 times with 5 bar $N_{2}$ and 6 bar $H_{2}$ to remove any traces of oxygen. The reactor is equipped with a PID controller to monitor and control temperature and agitation speed. A dip tube connected to a valve was used for withdrawal of liquid samples by the action of pressure difference. This procedure was carried out every 30 minutes. Approximately $2 \mathrm{ml}$ of sample were retrieved from the reactor into a $5 \mathrm{ml}$ vial which was put immediately in ice-water for several minutes and analyzed. The collection of samples in multiphase systems get a representative sample from the system. In this work, the only solution to this problem was to collect the sample using the sampling port, separate the two phases, measure each individually in weight and volume to verify mass balances, 
and then recombine them for further analysis. A scheme of the set-up used is depicted in Figure 2, 11 experiments were carried out based on a Box-Behnken design for three factors (temperature, oil-to-water ratio, and agitation speed) and an experimental runtime of 6 hours as shown in Table 1 [39.

Table 1: Experimental design (validation sets are highlighted)

\begin{tabular}{cccc}
\hline \multicolumn{4}{c}{ Experimental conditions } \\
\hline Experiment & Temperature $\left({ }^{\circ} \mathbf{C}\right)$ & Water-to-oil ratio & Agitation (rpm) \\
\hline $\mathbf{1}$ & $\mathbf{1 8 0}$ & $\mathbf{1 5}$ & $\mathbf{3 6 0}$ \\
$\mathbf{2}$ & $\mathbf{1 8 0}$ & $\mathbf{4 0}$ & $\mathbf{3 6 0}$ \\
3 & 280 & 15 & 360 \\
4 & 280 & 40 & 360 \\
5 & 180 & 27.5 & 120 \\
$\mathbf{6}$ & $\mathbf{1 8 0}$ & $\mathbf{2 7 . 5}$ & $\mathbf{6 0 0}$ \\
7 & 280 & 27.5 & 120 \\
8 & 280 & 27.5 & 600 \\
9 & 230 & 15 & 120 \\
10 & 230 & 15 & 600 \\
11 & 230 & 40 & 600 \\
\hline
\end{tabular}

The initial time of each experiment was defined as the point of time when oil and water reached the operating temperature (approximately 18 minutes).

\subsection{HPLC Analysis}

Collected samples were analyzed by following the procedure developed by [40, and modified by [41]. $40 \mu \mathrm{L}$ of the sample were injected in the HPLC (Ultimate 3000, Dionex A/S, Hvidovre, Denmark) to analyze TG, DG, MG, and FA. The separation of the compounds was performed in a cyanopropyl column $(0.25$ x 0.004 m) (Discovery, Cyano, Sigma Aldrich A/S, Brøndby, Denmark), U3000 auto-sampler, TCC-3000SD column oven, U3400A quaternary pump modules, and a Corona Charged Aerosol Detector (Thermo Scientific, MA, United States). A binary gradient program was run with the use of three solvents $(99.6 \% \mathrm{v} / \mathrm{v}$ tert-Butyl methyl ether, $0.4 \% \mathrm{v} / \mathrm{v}$ Acetic acid and, Isopropanol). The detection of the compounds was achieved by a Corona Charged Aerosol Detector from Thermo Scientific Dionex (Chelmsford, MA) with pressurized $\mathrm{N}_{2}$. 


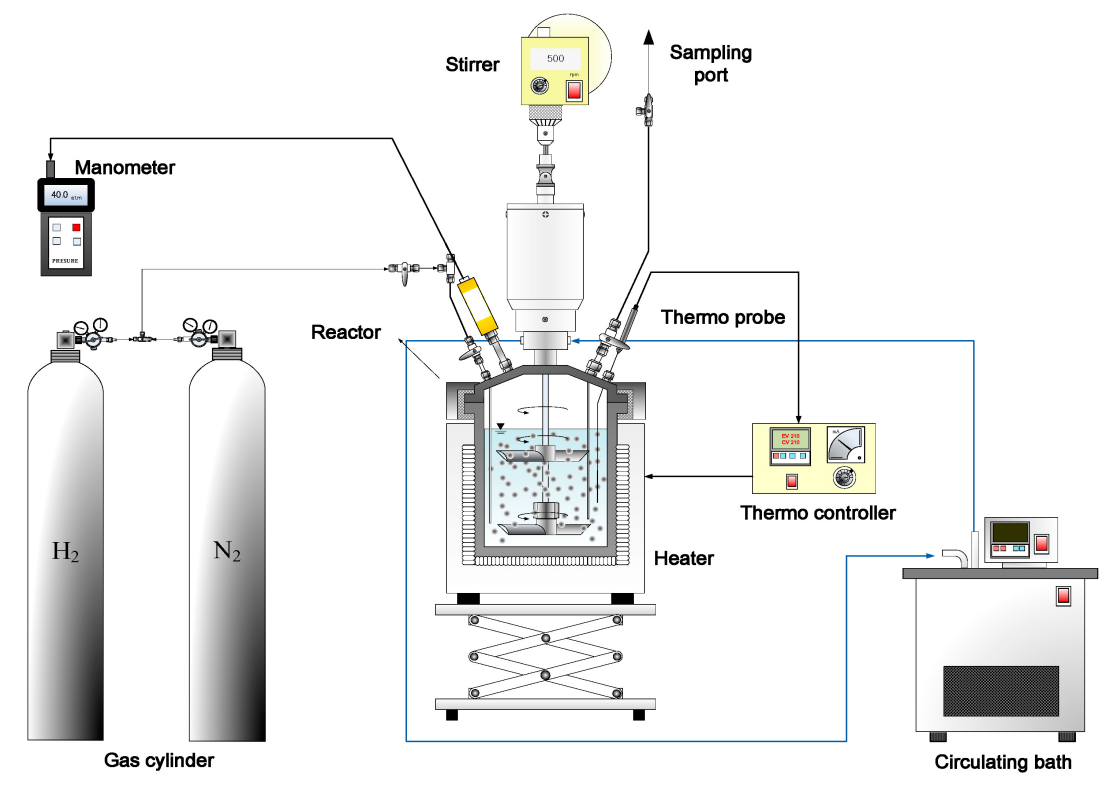

Figure 2: Reactor setup used for the hydrolysis of rapeseed oil

\section{Parameter estimation, uncertainty and sensitivity analysis}

\subsection{Sequential and simultaneous parameter estimation}

For the datasets collected in the experiments, kinetic and mass transfer parameters were estimated by applying the Levenberg-Marquardt algorithm for the non-linear global fitting problem. Thus, the Levenberg-Marquardt algorithm presents the simultaneously estimated parameters, the value of the residuals and the Jacobian matrix by minimizing the objective function in order to get the lowest sum of squared residuals for the kinetic and mass transfer parameters:

$$
\theta=\arg \min \sum_{i}\left(y_{i}^{\text {exp }}-y_{i}^{\text {pred }}\right)^{2}
$$

In Equation 200 $\theta$ are the estimated parameters, $y_{i}^{e x p}$ the measurement, and $y_{i}^{\text {pred }}$ the obtained value of parameter $i$.

This preliminary estimation gives an initial guess for the simultaneous estimation of parameters which is carried out as an ordinary non-linear regression problem. 
280

4.1.1. Covariance-based uncertainty analysis of parameter estimation and output prediction

The underlying assumption is that the errors are normally distributed. The uncertainty of the parameter estimates is based on the covariance matrix, $C O V(\theta)$, which is obtained through first linear approximation as described in the work285 flow proposed by [42]. The covariance matrix $\operatorname{COV}(\theta)$ is estimated by Equation 21):

$$
\operatorname{COV}(\theta)=\frac{S(\theta)}{N-p}\left(\left(\frac{d \mathbf{y}}{d \theta}\right)^{T} Q_{m}^{-1}\left(\frac{d \mathbf{y}}{d \theta}\right)\right)
$$

where $S(\theta)$ represents the minimum error sum of squares calculated from the Levenberg-Marquardt algorithm in the non-linear global fitting problem, $\frac{d y}{d \theta}$ is sensitivity matrix of the outputs obtained in the model $(\mathbf{y})$ with reference to $N$ is the number of experimental data used for the regression, and $p$ is the total number of estimated parameters. In this way, the correlation between two parameters $v$ and $w$ can be expressed as:

$$
\operatorname{COR}\left(\theta_{v}, \theta_{w}\right)=\frac{\operatorname{COV}\left(\theta_{v}, \theta_{w}\right)}{\sqrt{\sigma_{\theta_{v}}^{2} \sigma_{\theta_{w}}^{2}}}
$$

The histograms of residuals were used to verify that the residuals follow a 295 normal distribution and have a zero mean. This also allows one to evaluate the quality of the fitting by identifying the confidence regions for the parameters through the calculation of normal bivariate distributions.

\subsubsection{Monte Carlo method for uncertainty analysis of parameter estimation and prediction}

In this work, the calculation of the uncertainty in the obtained parameters was carried out by applying the Monte Carlo method. This technique uses randomly sampled parameters so as to evaluate the model outputs and to obtain their distribution. In this way, it is possible to achieve global results in the uncertainty due to the large number of evaluations the model undergoes. Thus, 
once the input parameter space is defined, the model is evaluated by obtaining the corresponding model outputs $\mathbf{y}$. The procedure for performing uncertainty analysis can be summarized as:

1. Definition of the input parameter uncertainty: upper and lower bounds for a model parameter are defined by its confidence intervals $\hat{\theta}$.

2. Sampling input uncertainty: quasi-random sampling of the parameter estimates by means of a Latin Hypercube Sampling to distribute samples evenly over the input parameter space 43 .

3. Use of the sampling matrix to evaluate the model: simulations are performed by using the sampled input matrix obtained in the previous step. In this way, a cumulative distribution function for each model output is obtained. Consequently, mean values of the model and $5^{t h}$ and $95^{t h}$ percentile calculations are used to represent the uncertainty of the model outputs.

\subsection{Variance based sensitivity analysis - Sobol method}

A variance based sensitivity analysis method uses the variance of the model outputs and decomposes it into fractions that can be related to the model parameters. These partial variances can be obtained by the decomposition of a random vector of input parameters. Then, the partial variances are normalized with the total variance to obtain the so-called Sobol sensivity indices [44].

The Sobol indices are useful to quantify the importance of a parameter $X_{i}$ on a model output. The Sobol indices can take values between 0 and 1 . In this method, a value closer to 1 means that the contribution of the input parameter on the variance is high. The Sobol sensitivity indices also allow one to identify the total and the interaction effect among parameters on the output variance for a specific input parameter [45].

The variance calculates measures on how far a set of random numbers are spread out from their mean value. The variance of a model output $y$ is given 
by:

$$
V(y)=V(f(X))=\sigma^{2}=\int(f(X)-\mu)^{2}-p(X) d X
$$

where $V(X)$ is the variance, $\sigma$ the standard deviation, $\mu$ the mean, $p(X)$ is the probability density function of $X$, and $X$ is a random parameter.

The variance can be decomposed by using Sobol's higher dimensional model representations as follows [46]:

$$
V(y)=\sum_{i} V_{i}+\sum_{i} \sum_{j>i} V_{i, j}
$$

where $V_{i}$ and $V_{i, j}$ are the first-order and second-order variance of the model outputs respectively.

Sobol indices can be obtained by normalizing the partial variances with the total variance as:

$$
S_{i}=\frac{V_{i}}{V(y)} \quad S_{i, j}=\frac{V_{i, j}}{V(y)}
$$

where $S_{i}$ is the first-order sensitivity index which allows one to characterize the influence of parameter $X_{i}$ on the model output. $S_{i, j}$ is the second-order sensitivity index, which allows the quantification of interactions among parameters ${ }_{345} X_{i}$ and $X_{j}$.

$S_{T i}$ is the total sensitivity index where the input parameter $X_{i}$ is present, which can be expressed as [4]:

$$
S_{T I}=S_{i}+\sum_{j} S_{i, j}
$$

These indices can be obtained by using Monte Carlo simulations (e.g. Janssen, Sobol, or Saltelli approximation) [48, 44, 49]. However, recently developed methodologies make use of metamodels, such as polynomial chaos expansions to overcome the computational costs related to the sampling of the Monte Carlo method. 


\subsubsection{Polynomial chaos expansions}

Polynomial chaos expansion (PCE) is a sampling-based method to determine orthogonal polynomials. The main advantage of representing the model output as a polynomial is that it allows one to simplify and speed-up the calculations required.

Different polynomial types can be used to approximate the model output. approximation depends on the type of distribution the input variable $X$ follows. For example, Legendre polynomials and Hermite polynomials are used for rectangular and standard normal distributions respectively. The model output can be approximated with different polynomial types. The approximated function is defined as:

$$
y=f(\mathbf{X}) \approx \sum_{a \in \mathbb{R}^{M}} \hat{y}_{a} \phi_{a}(\mathbf{X})
$$

where the coefficients $\hat{y}$ can be found by using Gauss-Legendre quadrature rules [50]:

$$
\hat{y}_{a} \approx \sum_{k=1}^{N}=f\left(x_{k}\right) \phi_{a}\left(x_{k}\right) w_{k}
$$

In Equation (28) $w_{k}$ represent the weights and $x_{k}$ the nodes, which can be calculated by the polynomial distribution function of the independent input parameters. Both the weights and nodes can be determined by finding the roots of the polynomial function [47.

Once this approximation of the model output is obtained, the total and partial variance of the function can directly be computed due to the orthogonality of the polynomials [47, 51]. Once the variances are obtained, the Sobol sensitivity indices can be computed.

$$
V_{t}=\sum_{\substack{a \in \mathbb{R}^{M} \\ a \neq 0}} \hat{y}_{a}^{2}
$$




$$
V_{i}=\sum_{\substack{a \in \mathbb{R}^{a} \\ a \neq 0}} \hat{y}_{a}^{2}
$$

$$
e=\mathbf{y}-\hat{\mathbf{y}}
$$

Where $e$ is a vector which contains the residuals, $y$ are the measurements taken in a experiment, and $\hat{y}$ is the output calculated in the model.

\section{Implementation and simulation environment}

The implementation of the methodologies, simulations, and programming of in this study [47, 59]. The implementation of the above presented methods is summarized in Table 2

\section{Results and discussion}

\subsection{Model fitting, parameter uncertainty and correlation}

395

Eight datasets obtained with the above described experimental procedure were used to estimate the model parameters through global fitting, while three 
Table 2: Uncertainty and sensitivity analysis method

\begin{tabular}{cccc}
\hline$\#$ & Step & Description & Output \\
\hline 1 & Parameter estimation & Parameters to fit the model & $\theta_{0}$ \\
& & Identification of parameter & $\hat{\theta}_{R}$ \\
& & Correlation matrix & $R_{\theta}$ \\
& & Confidence interval for parameters & $\sigma$ \\
\hline 2 & Uncertainty analysis & Prediction uncertainty of the model & $5^{\text {th }}$ and $95^{\text {th }}$ \\
\hline 3 & Sensitivity analysis & Sobol sensitivity indices & $S_{i}$ and $S_{T i}$ \\
& & Polynomial chaos expansions & $V_{i}$ and $V_{T}$ \\
\hline 4 & Residual analysis & Simulations with estimated parameters & \\
& & Probability distribution of residuals & \\
& & Compute the autocorrelation function & \\
\hline
\end{tabular}

are used for model validation. Model fits for the validation sets as well as the parameter estimation are presented in Figure 3 and Table 3 .

The performance of the model fits for the validation sets during the reaction times is shown in Figure 3, where data is provided in terms of moles of species. It can be seen that the quality of the model fits is high. The quality of the model fits and the experimental data is important in order to ensure that the model gives a true measure of the real system. In this regard, the Mean Absolute Error was quantified to measure the average magnitude of the errors in the predictions provided by the proposed model for the experimental validation sets. For the studied model outputs $n_{T G}, n_{D G}, n_{M G}$ and $n_{F A}$ the Mean Absolute error was 0.0042 moles, 0.0038 moles, 0.0037 moles and 0.0187 moles respectively, which can be considered as low. These fits were obtained by estimating 8 kinetic and 2 mass transfer parameters present in the developed mathematical model. Since

410 the estimation error obtained by the standard deviation is low, it is possible to say that the estimated values are accurate [54].

The proposed model captures the behaviour for the four analyzed component, although the prediction for $D G$ and $M G$ mismatches the experimental data. However in Figure 3 , the experimental amount of moles of validation set $4151\left(180^{\circ} \mathrm{C}-1: 15\right.$ oil-to-water molar ratio) shows significant mismatches, when it 
comes to the prediction of $M G$ which is much higher than the amount predicted by the model. The model mismatches observed can be related to changes in the phenomena which were not modeled such as viscosity and due to the large generation of emulsifying agents such as $D G$ and $M G$, which in addition to forming hydrogen bonds, can be combined in the mixture with small amounts of water and glycerol changing then the distribution of phases as reported by Wang et al 60]. At $180^{\circ} \mathrm{C}-1: 40$ oil-to-water molar ratio $-360 \mathrm{rpm}$, there is a higher amount of $F A$ produced which is in agreement with previous research, since it is known that high amounts of water lead to higher conversion rates [60, 12, 13].

The mean of the estimated parameters, their standard deviation $\sigma$, and correlation coefficients are shown in Table 3 Once parameter estimates are obtained, it is necessary to determine how specific they are in relation to the experimental data used. When two parameters are highly correlated, a change in the model output can be mitigated by a change in the value of the other parameter. As seen in Table 3, some of the parameters are highly correlated, which prevents to find a unique estimate of the parameter value.
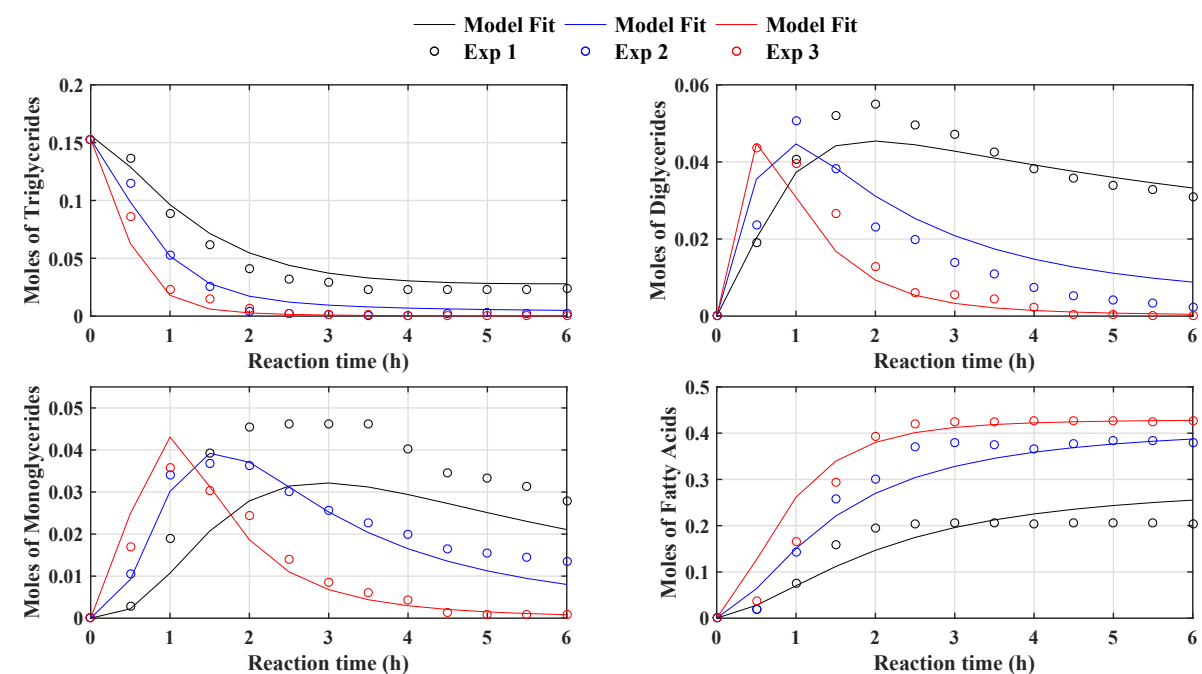

Figure 3: Global fitting and experimental data for the hydrolysis at (-) $180^{\circ} \mathrm{C}-1: 15$ oilto-water molar ratio - $360 \mathrm{rpm},(-) 180^{\circ} \mathrm{C}-1: 27.5$ oil-to-water molar ratio $-600 \mathrm{rpm}$ and (—) $180^{\circ} \mathrm{C}-1: 40$ oil-to-water molar ratio-360 rpm. 
Table 3: Mean, standard deviation and correlation matrix for the parameter estimation.

\begin{tabular}{ccccccccccccc}
\hline \multirow{2}{*}{$\theta$} & Mean & \multicolumn{1}{c}{ Correlation matrix } \\
& & & $k_{1}$ & $k_{-1}$ & $k_{2}$ & $k_{-2}$ & $k_{3}$ & $k_{-3}$ & $k_{4}$ & $k_{-4}$ & $k_{1 L, a}$ & $k_{2 L, a}$ \\
\hline$k_{1}$ & 2.029 & 0.101 & 1.000 & & & & & & & & & \\
$k_{-1}$ & 1.919 & 0.208 & 0.655 & 1.000 & & & & & & & & \\
$k_{2}$ & 2.251 & 0.203 & 0.112 & 0.013 & 1.000 & & & & & & & \\
$k_{-2}$ & 4.236 & 0.528 & 0.123 & -0.137 & 0.931 & 1.000 & & & & & & \\
$k_{3}$ & 1.173 & 0.057 & 0.059 & 0.168 & -0.079 & -0.059 & 1.000 & & & & & \\
$k_{-3}$ & 0.395 & 0.031 & -0.103 & -0.292 & -0.092 & -0.029 & 0.603 & 1.000 & & & & \\
$k_{4}$ & 2.464 & 0.128 & -0.138 & 0.096 & -0.111 & -0.185 & -0.001 & -0.155 & 1.000 & & & \\
$k_{-4}$ & 1.75 & 0.102 & -0.268 & -0.431 & -0.302 & -0.262 & -0.111 & 0.063 & 0.068 & 1.000 & & \\
$k_{1 L, a}$ & 0.319 & 0.025 & -0.773 & -0.424 & -0.524 & -0.499 & -0.063 & 0.061 & 0.191 & 0.292 & 1.000 & \\
$k_{2 L, a}$ & 0.772 & 0.13 & -0.273 & -0.222 & 0.063 & 0.182 & -0.426 & -0.242 & -0.336 & 0.054 & 0.255 & 1.000 \\
\hline
\end{tabular}

For example, $k_{1}$ has a high negative correlation with $k_{1 L, a}$, which means that the kinetic constant related to the consumption of triglycerides has a negative effect on the mass transfer coefficient of water. Our study also shows that if the value of $k_{1}$ increases, $k_{1 L, a}$ decreases to obtain a good fit. Similar analyses can be done for $k_{1}$ and $k_{-1}$ (consumption and generation of $T G$ in (Reaction 1) $), k_{-1}$ and $k_{-4}$ (consumption and generation of $D G$ in (Reaction 1) and (Reaction 4), $k_{2}$ and $k_{-2}$ (generation and consumption of $M G$ in (Reaction 2) $), k_{-2}$ and $k_{1 L, a}$ (consumption of $M G$ in (Reaction 2 and mass transfer of water respectively),

440 $k_{3}$ and $k_{-3}$ (generation and consumption of $M G$ ). This correlation is expected, since the hydrolysis proceeds as a set of parallel and sequential reactions, which implies poor identifiability. In this regard, more measurements should be performed so as to be included in the parameter estimation. These measurements can be moles of glycerol and water as well as separate measurements of mass transfer rates between phases without the complexity of ongoing reaction.

It is worth mentioning that the uncertainty results presented in this work are conditional to the range defined by the parameter uncertainty alongside their correlation coefficients. Therefore, the results need to be handled accordingly within those conditions [52. In this context, the results provided by the uncertainty analysis and parameter estimation are of local nature and are valid in the neighborhood at which the minimization was performed in the parameter estimation. To overcome the identifiability issues, it is then necessary to 
find a unique combination of estimated parameters which provide low correlation and low confidence intervals. However, the major disadvantage of this 455 approach is that those parameters that are found unidentifiable need to be estimated from independent experiments, which can be expensive. In order to generalize the results obtained, it is required to perform a sensitivity analysis based-identifiability analysis iteratively at different conditions or alternatively one could perform a global sensitivity analysis as the one carried out in this work for model improvement [61, 52]. Another valid option is to accept the model with its parameter uncertainty and use it to evaluate whether it can be applied in process engineering tasks as well as process design and optimization.

\subsection{Uncertainty analysis of model predictions}

The uncertainty of the calculated model outputs $\left(n_{T G}, n_{D G}, n_{M G}\right.$, and $\left.n_{F A}\right)$ during the hydrolysis reaction can be seen in Figure 4 and Figure 5 . In these figures, synthetic data generated by the evaluation of the model by using Latin Hypercube Samples is shown for the validation set (hydrolysis at $180^{\circ} \mathrm{C}-1: 27.5$ oil-to-water molar ratio - $600 \mathrm{rpm}$ ). It is possible to observe that almost all the experimental values lay inside the working boundaries of the simulations. The tight predictions for $n_{T G}$ and $n_{F A}$ give an overview of the robustness of the model and quality of the fit, while the large predictions calculated for $n_{D G}$ and $n_{M G}$ indicate that either the model which contains deficiencies or the variables were not properly measured. In this case, a better estimation of the parameters and more experiments are necessary to get more accurate predictions for these 475 species.

\subsection{Sensitivity analysis of model outputs}

For the sensitivity analysis with Sobol indices an output was needed, given our interest in determining which parameters contribute to the uncertainty of the model outputs during the time course of the reaction ( 0.5 - 6 hours), where there are significant variations in the model outputs of the Monte Carlo simulations (Figure 4). Global calculations were also made by using the mean values of 

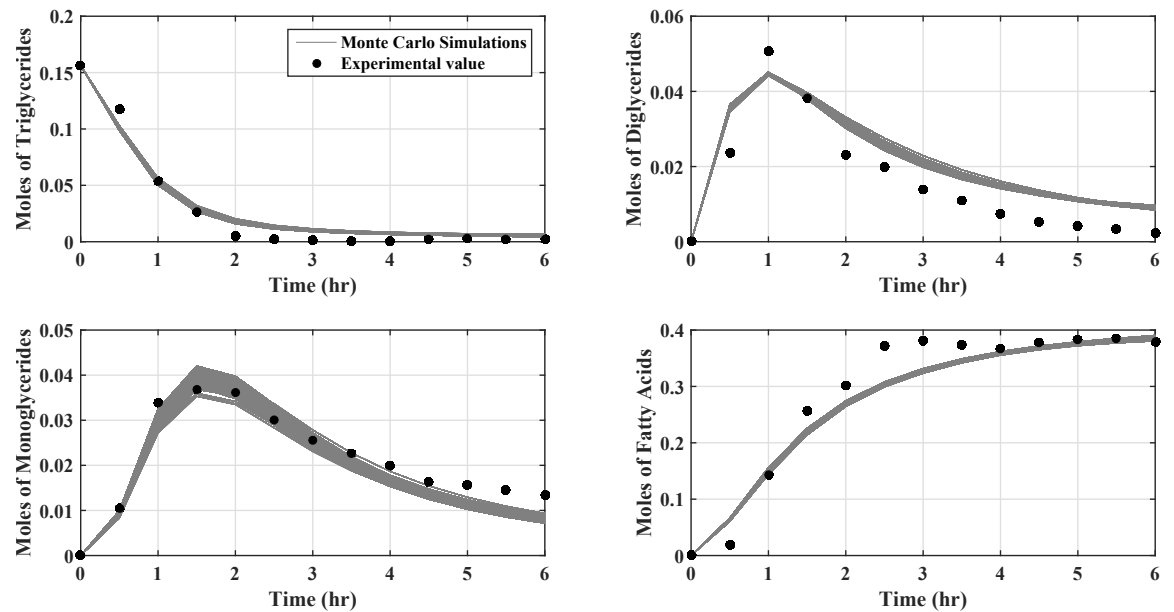

Figure 4: Uncertainty propagation generated from the simulations based on Monte Carlo method for the selected dataset (hydrolysis at $180^{\circ} \mathrm{C}-1: 27.5$ oil-to-water molar ratio - 600 rpm) (-)
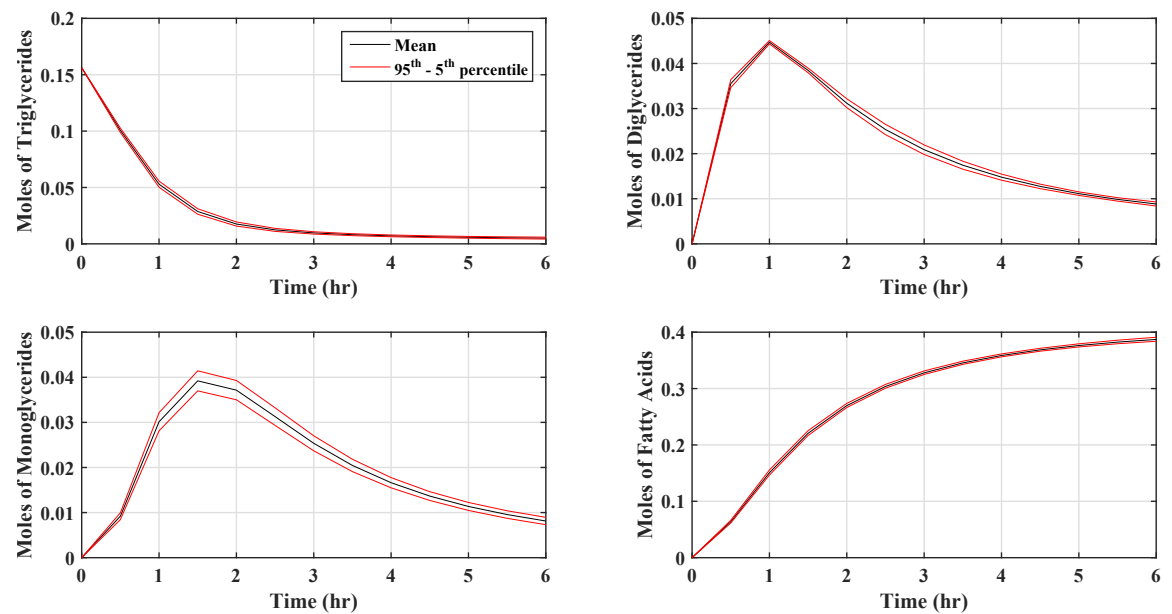

Figure 5: Uncertainty propagation represented by the mean (-) and the $5^{\text {th }}$ and $95^{\text {th }}$ percentiles (-) of the Monte Carlo simulations for the selected dataset (hydrolysis at $180^{\circ} \mathrm{C}$ - 1:27.5 oil-to-water molar ratio - $600 \mathrm{rpm}$ ) 
the outputs. This was done to rank the significance of the parameters obtained at the different conditions used in the validation sets. It should be noted that the analysis can be performed at different time points and different process 485 conditions at which case the parameter ranking can vary. In this case, the selected set of conditions was the hydrolysis at $180^{\circ} \mathrm{C}-1: 27.5$ oil-to-water ratio - $600 \mathrm{rpm}$.

Results for the variance-based sensitivity analysis are expressed by two sensitivity indices: the Sobol first order sensitivity indices $S_{i}$ indicate the importance of each parameter considered individually, while the total sensitivity indices $S_{T i}$ account for both the importance of individual parameters and interactions between parameter pairs. In this study, the values of $S_{T i}$ are similar to $S_{i}$.

The highest sum of $S_{T i}$ obtained for the input parameters of $n_{M G}$ (1.0059), as seen in Table 4 , shows that the variations in outputs are motivated by firstorder effects of the parameters, while the second-order interaction terms contribute to only $0.59 \%$ of the variance of the model output 62 (See also Supporting Information).

Through the analysis of the sensitivity analysis for the mean values of the validation set, it can be noticed that the consumption of $n_{T G}$ is most influenced by kinetic parameters $k_{1}$ and $k_{-1}$ in (Reaction 1 ) along with the mass transfer coefficient of water $k_{1 L, a}$. It is explained by the fact that the the mass transfer of water is bound to the reaction with $n_{T G}$. Hence, the resulting decrease or increase in mass transfer should then affect all the model outputs. For the parameters in the reactions related to the formation and consumption of $n_{D G}$, the most influential parameters are $k_{2}$ and $k_{-2}$ in (Reaction 2), while the remaining parameters are deemed irrelevant. In the case of $n_{M G}$, kinetic parameters $k_{3}$ and $k_{-3}$ in (Reaction 3) have the higher impact. Consequently, the most important parameters related to generation of the product $n_{F A}$ are the rate constants for forward reactions alongside the rate constants for reverse reactions (Reaction 1) 510 - Reaction 3). As seen in Table 4 , the parameters $k_{4}, k_{-4}$, and $k_{2 L, a}$ are little or non-influential for the model outputs coupled with the experimental data. Parameters $k_{4}, k_{-4}$ which are related to the fourth reaction step were included 
in the model to account for additional reactions which occur in the system with unreacted triglycerides and generated diglycerides. In this case, its low contribution to the output variance could indicate that such reaction is not taken place in the reactors. In the same way, low values for the mass transfer coefficient of glycerol $k_{2 L, a}$ could be related to a low generation of glycerol which therefore is not relevant in the mass transfer process. Therefore, values for these parameters can be fixed to any value within their confidence intervals without influencing the model outputs. Non-influential parameters contribute to a small percentage of the total variance, which then provide criteria for model simplification.

Table 4: Sensitivity analysis with first order Sobol' indices for each model parameter for the hydrolysis at $180^{\circ} \mathrm{C}-1: 27.5$ oil-to-water molar ratio - $600 \mathrm{rpm}$

\begin{tabular}{c|cc|cc|cc|cc}
\hline Rank & \multicolumn{2}{|c|}{ Triglycerides } & \multicolumn{2}{c|}{ Diglycerides } & \multicolumn{2}{c|}{ Monoglycerides } & \multicolumn{2}{c}{ Fatty acids } \\
\hline 1 & $k_{1}$ & 0.5476 & $k_{2}$ & 0.7355 & $k_{3}$ & 0.6547 & $k_{2}$ & 0.3735 \\
2 & $k_{-1}$ & 0.2339 & $k_{-2}$ & 0.2149 & $k_{-3}$ & 0.2185 & $k_{3}$ & 0.1778 \\
3 & $k_{1 L, a}$ & 0.1181 & $k_{3}$ & 0.0234 & $k_{2 L, a}$ & 0.0591 & $k_{1}$ & 0.1521 \\
4 & $k_{2}$ & 0.0671 & $k_{-3}$ & 0.0073 & $k_{2}$ & 0.0344 & $k_{-2}$ & 0.1096 \\
5 & $k_{-2}$ & 0.0327 & $k_{1 L, a}$ & 0.0068 & $k_{-2}$ & 0.0257 & $k_{-3}$ & 0.0622 \\
6 & $k_{-4}$ & 0.0009 & $k_{1}$ & 0.0051 & $k_{1 L, a}$ & 0.0049 & $k_{1 L, a}$ & 0.0553 \\
7 & $k_{3}$ & 0.0006 & $k_{-1}$ & 0.0045 & $k_{1}$ & 0.0043 & $k_{-1}$ & 0.055 \\
8 & $k_{-3}$ & 0.0004 & $k_{2 L, a}$ & 0.0019 & $k_{-1}$ & 0.0043 & $k_{2 L, a}$ & 0.0167 \\
9 & $k_{4}$ & 0.0002 & $k_{-4}$ & 0.0018 & $k_{4}$ & 0 & $k_{4}$ & 0 \\
10 & $k_{2 L, a}$ & 0.0001 & $k_{4}$ & 0.0004 & $k_{-4}$ & 0 & $k_{-4}$ & 0 \\
\hline \multicolumn{1}{c|}{$\sum S_{i}=0.9984$} & $\sum S_{i}=0.9984$ & $\sum S_{i}=0.9939$ & $\sum S_{i}=0.9978$ \\
\multicolumn{2}{c}{$\sum S_{T i}=1.0016$} & $\sum S_{T i}=1.0016$ & $\sum S_{T i}=1.0059$ & $\sum S_{T i}=1.0022$ \\
\hline
\end{tabular}

In Figure 6 and Figure 7, we show graphically the first order indices $S_{i}$ obtained by Sobol's method of the mass transfer phenomena $\left(k_{1 L, a}\right.$ and $\left.k_{2 L, a}\right)$ and the different reaction pathways $\left(k_{1}\right.$ and $k_{-1}, k_{2}$ and $k_{-2}, k_{3}$ and $k_{-3}$, and $k_{4}$ and $\left.k_{-4}\right)$ to the measured outputs $n_{T G}$ and $n_{F A}$ at different time steps. Detailed data concerning the sensitivity analysis of model outputs $n_{M G}$ and $n_{D G}$ is available in the Supporting Information. These indices give information about the significance of each parameter, where high values indicate higher significance and vice versa smaller values indicate negligible or no significance. Nonetheless, total sensitivity indices $S_{T i}$ give information related to the first- 
order effects of the parameters in the the model and the mutual interactions among parameters.

In Figure 6 and 7 , we present the global sensitivity analysis results conducted as the reaction progresses. The most important insight provided by these results and reversible. Hence, it is possible that every group of parameters might affect the equations which govern the model differently. When it comes to $n_{F A}$ in Figure 77 the main contributors to the sensitivity, at the beginning, are rate constant for forward (Reaction 1) and the mass transfer coefficient of water. oil phase bulk where the reaction takes place. Then, kinetic parameters related to (Reaction 1), (Reaction 2), and (Reaction 3) contribute greatly, which is explained by the fact that the mole balances involving $F A$ do not have a mass transfer component since the generated products are assumed to remain in the oil phase, where they were generated.

A closer look to the 10 estimated parameters shows that the mass trans- 


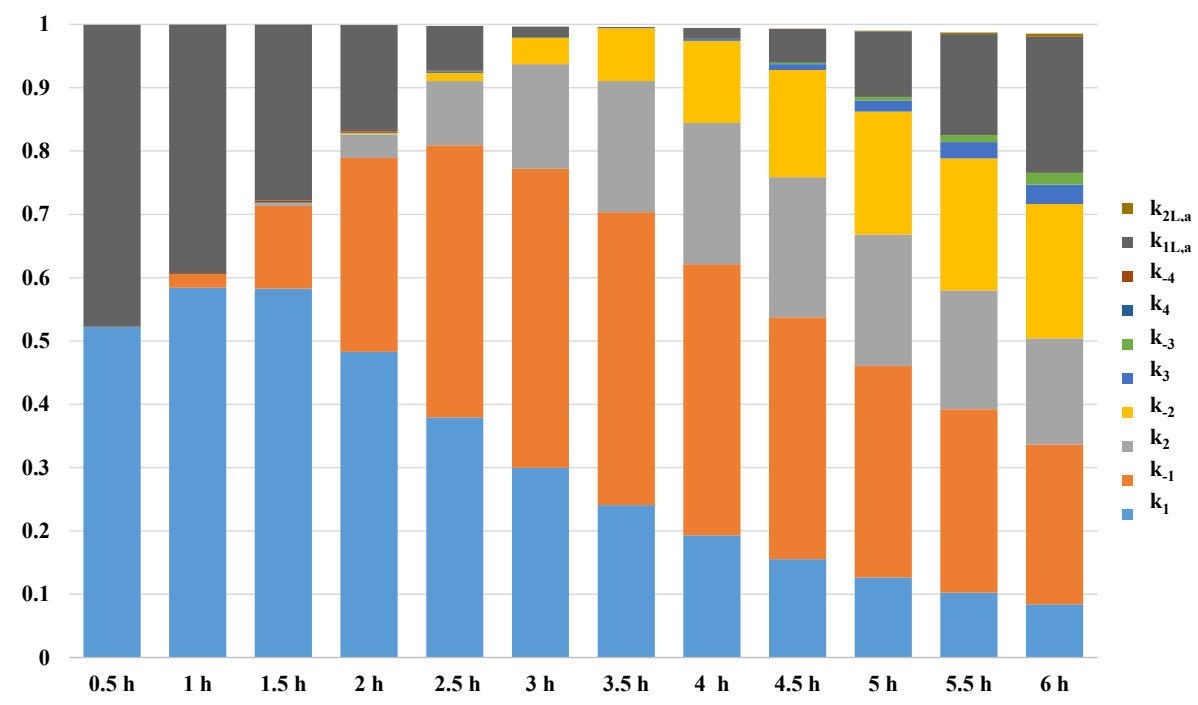

Figure 6: Contribution to the variations in the model output $n_{T G}$ over time using Sobol sensitivity indices for the hydrolysis at $180^{\circ} \mathrm{C}-1: 27.5$ oil-to-water molar ratio $-600 \mathrm{rpm}$

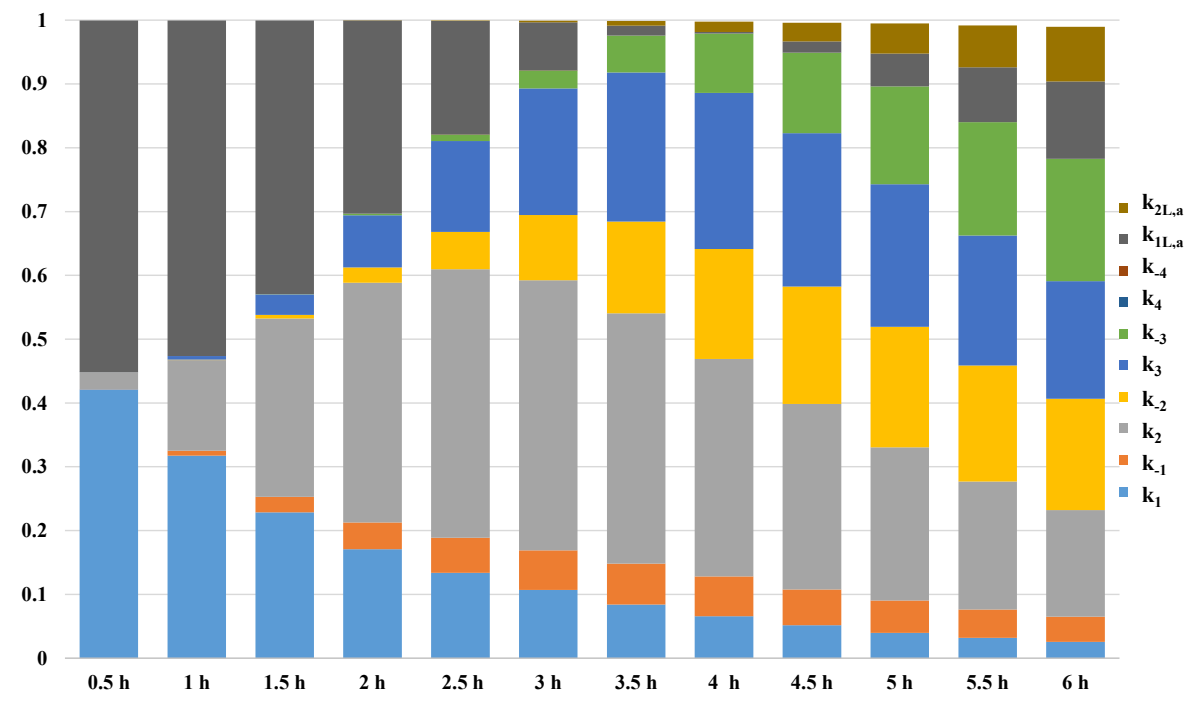

Figure 7: Contribution to the variations in the model output $n_{F A}$ over time using Sobol sensitivity indices for the hydrolysis at $180^{\circ} \mathrm{C}-1: 27.5$ oil-to-water molar ratio - $600 \mathrm{rpm}$ 
fer phenomena $\left(k_{1 L, a}\right.$ and $\left.k_{2 L, a}\right)$ and the three reaction pathways $\left(k_{1}\right.$ and $k_{-1}$ (Reaction 1), $k_{2}$ and $k_{-2}$ (Reaction 2), $k_{3}$ and $k_{-3}$ (Reaction 3) $)$, are the parameters which have a relatively significant effect on all the four model outputs. The non-influential parameters indicate that at the given experimental conditions, they do not affect the measured outputs. Therefore such information can be used as input to prioritize the research areas, for example, in improving the design of experiments to estimate those parameters or in simplifying the model. This is relevant in particular when reparametrizing and recalibrating the model for different feedstock with different initial composition (e.g. palm fatty acid distillate) or operating conditions (use of catalyst).

The results from the uncertainty analysis showed that the parameter estimates are trustworthy given the narrow working bounds obtained in the model outputs, which is of great interest for predictive purposes. It should be mentioned that the mass transfer coefficients can change during the reaction given the change of the component fractions in the consumed and generated phases. Hence, the values for these are deemed as average. In this regard, uncertainty analysis helped to validate the assumption that the estimated parameters are constant during the reaction. For process development, these analysis are useful when choosing type and finding optimal configurations in reactors. For example the use of batch data to predict reactor configuration residence times and conversions are easy to apply and fast to use. This analysis is therefore of considerable interest to improve the understanding of design and operating variables that increase the feasibility of vegetable oil utilization. It is because they provide information that could be used to compare different modeling approaches under different operating conditions.

\subsection{Analysis of residuals}

The residuals are calculated based on the validation set (hydrolysis at $180^{\circ} \mathrm{C}$, 1:27.5 oil-to-water mol ratio and $600 \mathrm{rpm}$ ) and are presented in Figure 8 The errors for $n_{T G}, n_{D G}, n_{M G}$, and $n_{F A}$ stay within -0.02 and 0.02 moles. If the residuals follow a standard normal distribution and are uncorrelated, the use of 
the model does not yield to errors in the evaluations. The Gaussian probability plots in Figure 8 shows the distance of the residuals to a standard distribution. The third plot in Figure 8 determines if there is any information in the residuals that is not obtained when the model is evaluated. It can be seen that there are spikes in almost all plots, however, they are not significant because they do not exceed the limit set by the confidence intervals.
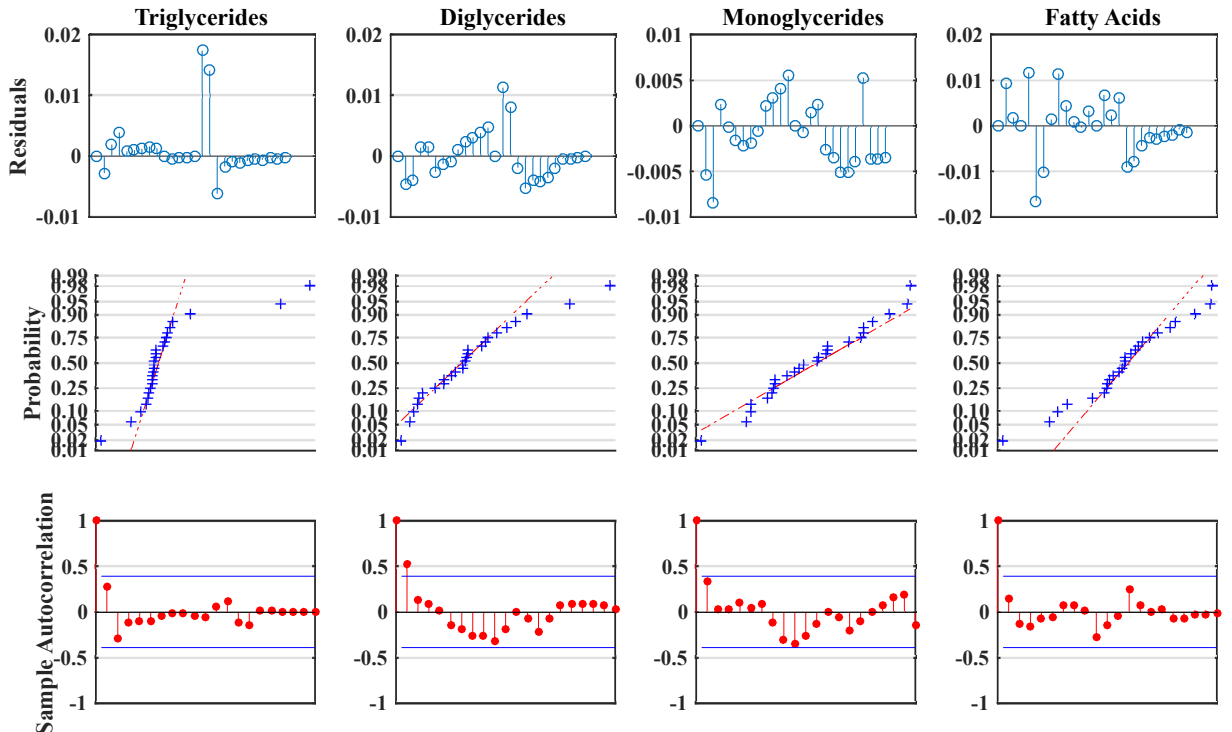

Figure 8: Residual analysis for the validation set (hydrolysis at $180^{\circ} \mathrm{C}, 1: 27.5$ oil-to-water mol ratio and $600 \mathrm{rpm}$ )

\section{Conclusions}

In this work a model for the hydrolysis of vegetable oils in a batch reactor un-

600 der subcritical conditions was developed and the parameters in the model were estimated from experimental data. The model includes mass transfer between oil and water phase and kinetics of the reversible hydrolysis reactions. The parameter estimation results showed that, while the parameter estimates were accurate, the pairwise correlation between estimates were significant for some parameters. This indicates that the available experimental data is not sufficient 
to uniquely identify the mass and kinetic parameters and thus requires further and improved experiment design. A Monte Carlo based uncertainty analysis was performed to find the accuracy of estimated parameters, the mean and standard deviations of the model outputs from experimental data. This method was then successfully applied to calculate the accuracy of the estimated parameters as well as their confidence intervals. We recommend this procedure for the estimation of the variance of parameters in chemical kinetics modeling. The results showed that the presented model was able to predict accurately the experimental data with a narrow confidence interval. The performed sensitivity analysis detected the influential and non-influential parameters to the model outputs, which allows to validate the model and assumptions proposed. This analysis provided insights on the relative importance of parameters and their changes as the reaction progresses, being the parameters related to the mass transfer $\left(k_{1 L, a}\right.$ and $\left.k_{2 L, a}\right)$ and first steps of the reacting system linked to the consumption of triglycerides the most important ( $k_{1}$ and $k_{-1}$ (Reaction 1) $)$. Since the lack of experimental data is a crucial issue in the hydrolysis of vegetable oils, this model-based analysis of data is of substantial value to provide necessary information for detailed modeling and characterization of the subcritical hydrolysis process.

\section{Acknowledgements}

This project has received funding from the European Union's Horizon 2020 research and innovation programme under the Marie Skłodowska-Curie grant agreement No. 675251.

\section{Nomenclature}

\section{Symbols}

$C_{i} \quad$ Concentration of component $i\left[\begin{array}{lll}m o l & L^{-1}\end{array}\right]$

$D G \quad$ Diglycerides 
$F A \quad$ Fatty acids

Gly Glycerol

$635 \quad \mathrm{H}_{2} \mathrm{O}$ Water

$J_{i}^{j} \quad$ Mass transfer rate of component $i$ in phase $j\left[\right.$ mol $\left.L^{-1} h r^{-1}\right]$

$k_{i} \quad$ Rate constant for forward reaction $i\left[L \mathrm{~mol}^{-1} \mathrm{hr}^{-1}\right]$

$k_{-i} \quad$ Rate constant for reverse reaction $i\left[L \mathrm{~mol}^{-1} \mathrm{hr}^{-1}\right]$

$k_{1 L, a} \quad$ Volumetric mass transfer coefficient of water $\left[h r^{-1}\right]$

${ }_{640} k_{2 L, a} \quad$ Volumetric mass transfer coefficient of glycerol $\left[h r^{-1}\right]$

$m_{i} \quad$ Partition coefficient of component $i$

$M G \quad$ Monoglycerides

$n_{i}^{j} \quad$ Moles of component $i$ in phase $j[\mathrm{~mol}]$

$R_{i} \quad$ Reaction rate $\left[\mathrm{mol} L^{-1} h r^{-1}\right]$

${ }_{645} \quad t \quad$ Time $[h r]$

TG Triglycerides

$V_{j} \quad$ Volume of phase $j[L]$

\section{Greek letters}

$\rho_{i} \quad$ Molar volume of component $i\left[\begin{array}{lll}m o l & L^{-1}\end{array}\right]$

650 $\theta \quad$ Estimated parameter

\section{Subscripts and superscripts}

* Interface

$a q \quad$ Aqueous phase

$i \quad$ Component $1,2,3, . ., n$ 
$655 \quad$ Phase

oil Oil phase

\section{References}

\section{References}

[1] G. R. List, Forecasts of oilseed and vegetable oil production, Lipid Technology 29 (9-10) (2017) 107. doi:10.1002/lite.201700028

[2] J. O. Metzger, Fats and oils as renewable feedstock for chemistry, European Journal of Lipid Science and Technology 111 (9) (2009) 865-876. doi: 10.1002/ejlt.200900130.

[3] R. Höfer, J. Bigorra, Biomass-based green chemistry: sustainable solutions for modern economies doi:10.1080/17518250802342519.

[4] W. Hamm, R. J. Hamilton, G. Calliauw, Edible oil processing, Wiley, 2013.

[5] The Soap and Detergent Association - Glycerine \& Oleochemical Division,

口 Glycerine: an overview of terms, technical data, properties and performance, Tech. rep. (1990).

670 URL https://goo.gl/YaYzyX

[6] A. Sturzenegger, H. Sturm, Hydrolysis of Fats at High Temperatures, Industrial \& Engineering Chemistry 43 (2) (1951) 510-515. doi:10.1021/ ie50494a054.

[7] I. M. Noor, M. Hasan, K. B. Ramachandran, Effect of operating variables 675 on the hydrolysis rate of palm oil by lipase, Tech. Rep. 1 (2003). doi: 10.1016/S0032-9592(02)00263-7.

[8] G. Pugazhenthi, A. Kumar, Enzyme membrane reactor for hydrolysis of olive oil using lipase immobilized on modified PMMA composite mem-

』 brane, Journal of Membrane Science 228 (2004) 187-197. doi:10.1016/j. memsci.2003.10.007. 
[9] A. E. Bailey, F. Shahidi, Bailey's industrial oil and fat products, John Wiley \& Sons, 2005.

[10] BCC Research, Global Markets for Oleochemical Fatty Acids Use, Tech. rep., BCC Research (2013). URL http://bit.1y/2Imxc2p

[11] L. Hartman, Kinetics of the Twitchell Hydrolysis, Nature 167 (4240) (1951) 199-199. doi:10.1038/167199a0.

[12] E. Minami, S. Saka, Kinetics of hydrolysis and methyl esterification for biodiesel production in two-step supercritical methanol process, Fuel 85 (17-18) (2006) 2479-2483. doi:10.1016/j.fuel.2006.04.017

[13] T. A. Patil, T. S. Raghunathan, H. S. Shankar, Thermal hydrolysis of vegetable oils and fats. 2. Hydrolysis in continuous stirred tank reactor, Industrial and Engineering Chemistry Research 27 (5) (1988) 735-739. doi: 10.1021/ie00077a002

${ }_{695}$ [14] T. A. Patil, T. S. Raghunathan, H. S. Shankar, Thermal hydrolysis of vegetable oils and fats. 2. Hydrolysis in continuous stirred tank reactor,

【 Industrial and Engineering Chemistry Research 27 (5) (1988) 735-739. doi: 10.1021/ie00077a002

[15] P. D. Namdev, T. A. Patil, T. S. Raghunathan, H. S. Shankar, Thermal

700 hydrolysis of vegetable oils and fats. 3. An analysis of design alternatives,

【 Industrial \& Engineering Chemistry Research 27 (5) (1988) 739-743. doi: 10.1021/ie00077a003.

[16] N. Sonntag, Fat splitting, Journal of the American Oil Chemists' Society 56 (11Part1) (1979) 729A-732A.

705 [17] I. M. Hill, Countercurrent hydrolysis of fat, uS Patent 2,480,471 (Aug. 30 1949). 
[18] J. A. Kent, et al., Kent and Riegel's handbook of industrial chemistry and biotechnology, Vol. 1665, Springer, 2007.

[19] L. Lascaray, Industrial fat splitting, Journal of the American Oil Chemist' Society 29 (9) (1952) 362-366. doi:10.1007/BF02631459

[20] L. Lascaray, Mechanism of Fat Splitting, Industrial \& Engineering Chemistry 41 (4) (1949) 786-790. doi:10.1021/ie50472a025

[21] R. Alenezi, G. Leeke, R. Santos, A. Khan, Hydrolysis kinetics of sunflower oil under subcritical water conditions, Chemical Engineering Research and Design 87 (6) (2009) 867-873. doi:10.1016/j.cherd.2008.12.009.

[22] J. K. Satyarthi, D. Srinivas, P. Ratnasamy, Hydrolysis of vegetable oils and fats to fatty acids over solid acid catalysts, Applied Catalysis A: General 391 (1-2) (2011) 427-435. doi:10.1016/j .apcata.2010.03.047.

[23] J. O. Metzger, U. Bornscheuer, Lipids as renewable resources: current state of chemical and biotechnological conversion and diversification, Appl Microbiol Biotechnol 71 (2006) 13-22. doi:10.1007/s00253-006-0335-4.

[24] J. Frutiger, M. Jones, N. G. Ince, G. Sin, From property uncertainties to process simulation uncertainties-monte carlo methods in simsci pro/ii process simulator, in: Computer Aided Chemical Engineering, Vol. 44, Elsevier, 2018, pp. 1489-1494.

[25] C. E. Goering, A. W. Schwab, M. J. Daugherty, E. H. Pryde, A. J. Heakin, Fuel properties of eleven vegetable oils, Transactions of the ASAE 25 (6) (1982) 1472-1477. doi:10.13031/2013.33748.

[26] H. Noureddini, D. W. Harkey, M. R. Gutsman, A continuous process for the glycerolysis of soybean oil, Journal of the American Oil Chemists' Society 81 (2) (2004) 203-207. doi:10.1007/s11746-004-0882-y.

[27] G. Astarita, Mass transfer with chemical reaction, 1967. 
[28] P. Trambouze, H. Van Landeghem, J. P. Wauquier, Chemical Reactors: Design, Engineering, Operation, Chemical Engineering Science 44 (7) (1989) 1599. doi : 10.1016/0009-2509(89)80040-5

[29] M. Attarakih, T. Albaraghthi, M. Abu-Khader, Z. Al-Hamamre, H.-J. Bart, Mathematical modeling of high-pressure oil-splitting reactor using a reduced population balance model, Chemical Engineering Science 84 (2012) 276-291. doi:10.1016/j.ces.2012.08.046.

[30] W. G. Whitman, The two film theory of gas absorption, International 1 Journal of Heat and Mass Transfer 5 (5) (1962) 429-433. doi:10.1016/ 0017-9310(62) 90032-7.

[31] J. Lohmann, J. Gmehling, Modified UNIFAC (Dortmund). Reliable Model for the Development of Thermal Separation Processes., Journal of Chemical Engineering of Japan 34 (1) (2001) 43-54. doi:10.1252/jcej.34.43.

[32] J. Gmehling, J. Li, M. Schiller, A modified UNIFAC model. 2. Present parameter matrix and results for different thermodynamic properties, In-

प dustrial \& Engineering Chemistry Research 32 (1) (1993) 178-193. doi: 10.1021/ie00013a024.

[33] J. Gmehling, J. Lohmann, A. Jakob, J. Li, R. Joh, A Modified UNIFAC (Dortmund) Model. 3. Revision and Extension, Industrial \& Engineering Chemistry Research 37 (12) (1998) 4876-4882. doi:10.1021/ie980347z.

[34] J. Gmehling, R. Wittig, J. Lohmann, R. Joh, A Modified UNIFAC (Dortmund) Model. 4. Revision and Extension, Industrial \& Engineering Chemistry Research 41 (6) (2002) 1678-1688. doi:10.1021/ie0108043.

[35] A. Jakob, H. Grensemann, J. Lohmann, J. Gmehling, Further Development of Modified UNIFAC (Dortmund): Revision and Extension 5, Industrial \& Engineering Chemistry Research 45 (23) (2006) 7924-7933. doi:10.1021/ ie $060355 \mathrm{c}$. 
[38] C. A. Diaz-Tovar, R. Gani, B. Sarup, Computer-Aided Modeling of Lipid Processing Technology, Phd thesis, Technical University of Denmark (2011).

[39] G. E. P. Box, D. W. Behnken, Some New Three Level Designs for the 770 . Study of Quantitative Variables, Technometrics 2 (4) (1960) 455. doi: $10.2307 / 1266454$.

[40] T. A. Foglia, K. C. Jones, Quantitation of Neutral Lipid Mixtures Using High Performance Liquid Chromatography with Light Scattering Detection†, Journal of Liquid Chromatography \& Related Technologies 20 (12) (1997) 1829-1838. doi:10.1080/10826079708005545.

[41] J. Price, B. Hofmann, V. T. L. Silva, M. Nordblad, J. M. Woodley, J. K. Huusom, Mechanistic modeling of biodiesel production using a liquid lipase

[42] G. Sin, A. S. Meyer, K. V. Gernaey, Assessing reliability of cellulose hydrolysis models to support biofuel process design-Identifiability and uncertainty analysis, Computers and Chemical Engineering 34 (9) (2010) 1385-1392. doi:10.1016/j.compchemeng.2010.02.012

[43] R. L. Iman, W. J. Conover, A distribution-free approach to inducing rank correlation among input variables, Communications in Statistics

1 - Simulation and Computation 11 (3) (1982) 311-334. doi:10.1080/ 03610918208812265 . 
[44] I. M. Sobol, Sensitivity estimates for nonlinear mathematical models, Mathematical modelling and computational experiments 1 (4) (1993) 407-414.

[45] J. Klinkert, The characterization of uncertainty for steady state multiphase flow models in pipelines, Master's thesis, Delft University of Technology (2018).

[46] R. C. Smith, Uncertainty quantification: theory, implementation, and applications, Vol. 12, Siam, 2013.

[47] S. Marelli, C. Lamas, K. Konakli, C. Mylonas, P. Wiederkehr, B. Sudret, UQLab user manual - Sensitivity analysis, Tech. rep., Chair of Risk, Safety \& Uncertainty Quantification, ETH Zürich, report \# UQLab-V1.2106 (2019).

[48] M. J. Jansen, Analysis of variance designs for model output, Computer Physics Communications 117 (1-2) (1999) 35-43.

[49] A. Saltelli, P. Annoni, I. Azzini, F. Campolongo, M. Ratto, S. Tarantola, Variance based sensitivity analysis of model output. design and estimator for the total sensitivity index, Computer Physics Communications 181 (2) (2010) 259-270.

[50] F. B. Hildebrand, Introduction to numerical analysis, Courier Corporation, 1987.

[51] R. Al, C. R. Behera, A. Zubov, K. V. Gernaey, G. Sin, Meta-modeling based efficient global sensitivity analysis for wastewater treatment plants an application to the bsm2 model, Computers and Chemical Engineering 127 (2019) 233-246. doi:10.1016/j.compchemeng.2019.05.015.

[52] G. Sin, K. V. Gernaey, A. E. Lantz, Good modeling practice for PAT applications: Propagation of input uncertainty and sensitivity analysis, Biotechnology Progress 25 (4) (2009) 1043-1053. doi:10.1002/btpr.166 
[53] N. H. Azim, A. Subki, Z. N. B. Yusof, Abiotic stresses induce total phenolic, total flavonoid and antioxidant properties in Malaysian indigenous microalgae and cyanobacterium (2018). doi:10.1017/CB09781107415324.004.

[54] G. Sin, K. V. Gernaey, Data Handling and Parameter Estimation, in: Experimental Methods in Wastewater Treatment, IWA Publishing, 2016, pp. 201-234. doi:10.1017/CB09781107415324.004. in MATLAB and Simulink, SIAM Review 41 (3) (1999) 538-552. doi: $10.1137 / \mathrm{S} 003614459933425 \mathrm{X}$

[56] The Mathworks Inc., MATLAB and Statistics Toolbox, version 2016b (2016).

[57] L. F. Shampine, M. W. Reichelt, The MATLAB ODE Suite, Tech. rep. URL http://bit.1y/2Uatvzq

[58] T. F. Coleman, Y. Li, An Interior Trust Region Approach for Nonlinear Minimization Subject to Bounds.

URL https://ecommons . cornell . edu/handle/1813/6108

[59] R. Al, C. R. Behera, A. Zubov, G. Sin, Systematic framework development for the construction of surrogate models for wastewater treatment plants, Computer-aided Chemical Engineering (2018) 1909-1914doi: 10.1016/B978-0-444-64241-7.50313-X.

[60] W. C. Wang, R. H. Natelson, L. F. Stikeleather, W. L. Roberts, Product sampling during transient continuous countercurrent hydrolysis of canola oil and development of a kinetic model, Computers and Chemical Engineering 58 (2013) 144-155. doi:10.1016/j.compchemeng.2013.06.003.

[61] R. Brun, M. Kühni, H. Siegrist, W. Gujer, P. Reichert, Practical identifiability of ASM2d parameters - systematic selection and tuning of param840 \ eter subsets, Water Research 36 (16) (2002) 4113-4127. doi:10.1016/ S0043-1354(02) 00104-5. 
[62] A. Zubov, G. Sin, Multiscale modeling of poly (lactic acid) production: From reaction conditions to rheology of polymer melt, Chemical Engineering Journal 336 (2018) 361-375.

${ }_{845}$ [63] J. P. O'Connell, J. M. Haile, Thermodynamics: Fundamentals for applications, Vol. 9780521582, Cambridge University Press, Cambridge, 2005. doi:10.1017/CB09780511840234.

\section{Supporting Information}

\section{Liquid-Liquid Equilibrium Calculations}

Modified UNIFAC (Dortmund)

The modified UNIFAC (Dortmund) model has a combinatorial contribution $\left(\ln \gamma_{i}^{C}\right)$ to the activity coefficient, which is directly related to differences in size and shape of the molecules, and a residual contribution $\left(\ln \gamma_{i}^{R}\right)$ to define the energetic interactions between the molecules as presented by Gmehling et al [31, 32, 33, 34, 35].

$$
\ln \gamma_{i}=\ln \gamma_{i}^{C}+\ln \gamma_{i}^{R}
$$

The combinatorial part is given by:

$$
\ln \gamma_{i}^{\prime}=1-V_{i}^{\prime}+\ln V_{i}^{\prime}-5 q_{i}\left[1-\frac{V_{i}}{F_{i}}+\ln \left(\frac{V_{i}}{F_{i}}\right)\right]
$$

The pure-component parameters $r_{i}$ and $q_{i}$ are respectively, related to molecular van der Waals volume and molecular surface area. They are calculated as the sum of the group volume and group parameters, $R_{K}$ and $Q_{K}$.

The mole fraction of component $j$ in the mixture is denoted as $z_{j}$. Thus:

$$
r_{i}=\sum_{k} v_{k}^{(i)} R_{K} \quad q_{i}=\sum_{k} v_{k}^{(i)} Q_{K}
$$

Where $v_{k}^{i}$, always an integer, is the number of groups of type $k$ in the molecule $i$. The group parameters $R_{K}$ and $Q_{K}$ are normally obtained from van der Waals group volumes and surface areas. 
The residual part is given by:

$$
\ln \gamma_{i}^{R}=\sum_{k} v_{k}^{(i)}\left[\ln \Gamma_{k}-\ln \Gamma_{k}^{(i)}\right]
$$

${ }_{865} \Gamma_{k}$ is the group residual activity coefficient, and $\Gamma_{k}^{(i)}$ is the residual activity coefficient of group $k$ in a reference solution containing only molecules of type $i$.

$$
\begin{gathered}
\ln \Gamma_{k}=Q_{k}\left[1-\ln \left(\sum_{m} \theta_{m} \psi_{m k}\right)-\sum_{m} \frac{\left(\theta_{m} \psi_{m k}\right)}{\sum_{n} \theta_{n} \psi_{n k}}\right] \\
\theta_{m}=\frac{Q_{m} X_{m}}{\sum_{n} Q_{n} X_{n}} \quad X_{m}=\frac{\sum_{i} v_{m}^{(i)} x_{i}}{\sum_{i} \sum_{m} v_{k}^{(i)} x_{i}}
\end{gathered}
$$

The surface area fraction of group $m$ in the mixture is represented by $\theta_{m}$ and $X_{m}$ is the mole fraction of group $\mathrm{m}$ in the mixture. The group interaction parameter $\psi_{n m}$ characterizes the interaction between groups $m$ and $n$ at temperature $T$ through parameters $a, b$ and $c$.

$$
\Psi_{n m}=\exp \left(-\frac{a_{n m}+b_{n m} T+c_{n m} T^{2}}{T}\right)
$$

\section{Liquid-Liquid Equilibrium Algorithm}

The equilibrium for a liquid - liquid system is defined by the equation:

$$
\gamma_{i}^{\alpha} z_{i}^{\alpha}=\gamma_{i}^{\beta} z_{i}^{\beta} \quad i=1,2,3 \ldots, \text { Component }
$$

Where $\gamma_{i}$, the activity coefficient of component $i$ in phase $(\alpha$ or $\beta)$, is predicted using the modified UNIFAC (Dortmund) model. $z_{i}^{\alpha}$ and $z_{i}^{\beta}$ represent the mole fraction of component $i$ in phase $\alpha$ and $\beta$ respectively.

The algorithm is summarized as a flow diagram in Figure A.1 as proposed and presented by O'Connell and Haile [63. If the system temperature and pressure are known (as they usually are for liquid-liquid equilibrium situations), then the problem can be posed as an analogy to isothermal flash calculations. 
In such an approach, the known quantities are temperature $T$, pressure $P$ and the set of overall system mole fractions $z$. The following relation can be defined:

$$
z_{i}=\frac{n_{i}}{n_{\text {Total }}}=\frac{n_{i}^{\alpha}+n_{i}^{\beta}}{n_{\text {Total }}^{\alpha}+n_{\text {Total }}^{\beta}}
$$

Then the quantities to be computed would be the mole fractions in each phase, $z^{\alpha}$ and $z^{\beta}$, and the fraction of total material in one phase, $R=\frac{n_{i}^{\beta}}{n_{\text {Total }}}$.

Distributions coefficients for each component $m_{i}$ are defined by $m_{i}=\frac{z_{i}^{\beta}}{z_{i}^{\alpha}}$. In order to calculate the required mole fractions, a gamma-gamma formulation for the phase equilibrium equations needs to be expressed in terms of the distribution coefficients as:

$$
m_{i}=\frac{z_{i}^{\beta}}{z_{i}^{\alpha}}=\frac{\gamma_{i}^{\alpha}}{\gamma_{i}^{\beta}} \quad i=1,2, \ldots, \text { Component }
$$

For each component $i$ in the liquid-liquid system, a material balance is written as:

$$
z_{i}^{\alpha}(1-R)+z_{i}^{\beta} R=z_{i}
$$

Where $R=\frac{n_{i}^{\beta}}{n_{\text {Total }}}$. Then, by using Equation A.10 for the distribution coefficients, it is possible to eliminate $z_{i}^{\beta}$ in favor of $z_{i}^{\alpha}$ :

$$
z_{i}^{\alpha}(1-R)+z_{i}^{\alpha} m_{i} R=z_{i}
$$

Solving for $z_{i}^{\alpha}$ and $z_{i}^{\beta}$ :

$$
z_{i}^{\alpha}=\frac{z_{i}}{1+R\left(m_{i}-1\right)} \quad z_{i}^{\beta}=\frac{z_{i} m_{i}}{1+R\left(m_{i}-1\right)}
$$

However, the mole fractions in each phase must sum to unity, therefore, a Rachford-Rice type function $F$ must be defined as [37]:

$$
F \equiv \sum_{i}^{m} z_{i}^{\beta}-\sum_{i}^{m} z_{i}^{\alpha}=\sum_{i}^{m} \frac{z_{i}\left(m_{i}-1\right)}{1+R\left(m_{i}-1\right)}=0
$$

895

This Rachford-Rice type function is one equation in the unknown $R$, independent of the number of components present, which readily lends itself to a 
solution by Newton's method. A flow diagram for solving this problem is shown in Figure A.1

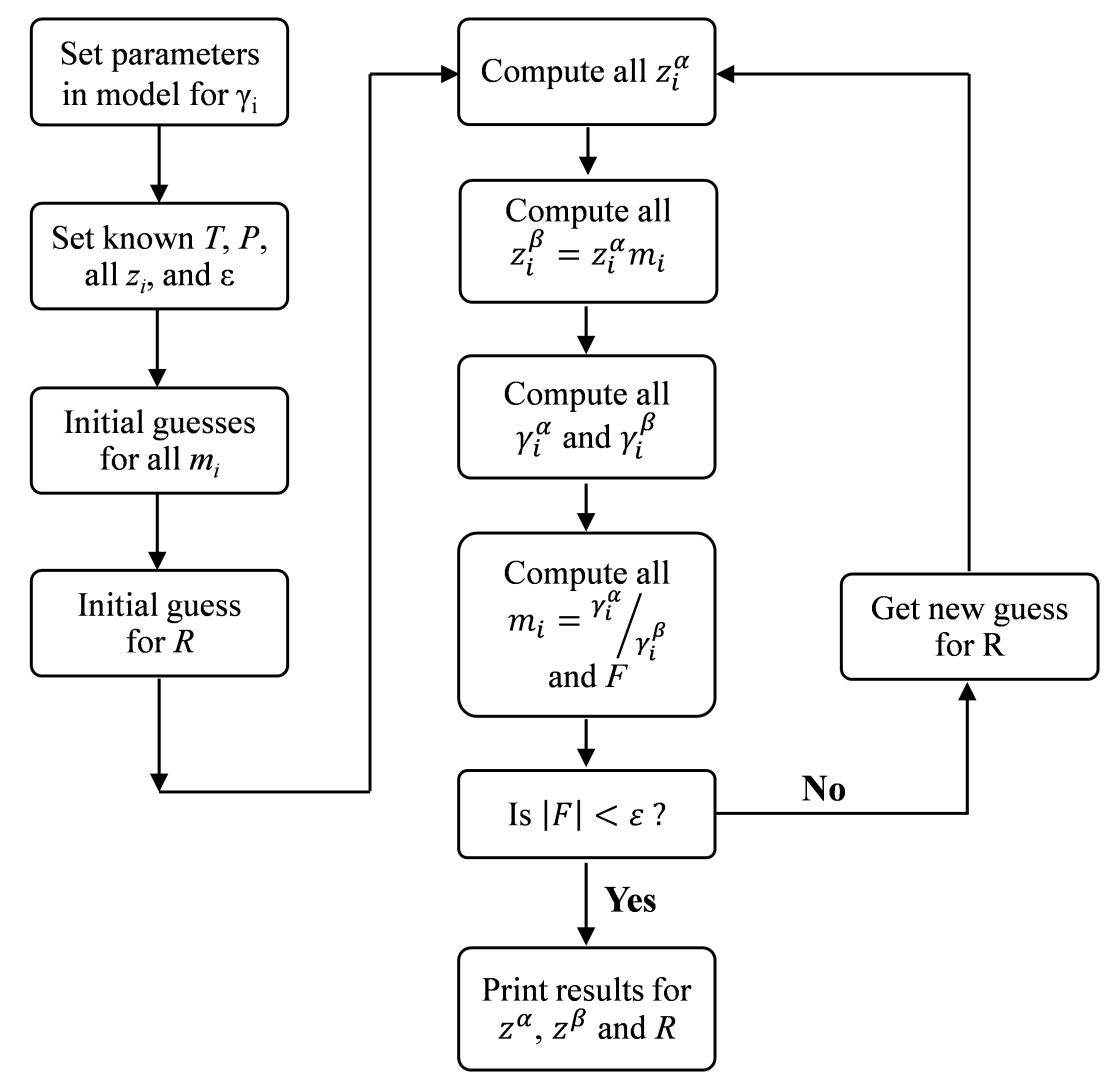

Figure A.1: Rachford-Rice algorithm applied to the gamma-gamma method for solving multicomponent liquid-liquid equilibrium problems (adapted from O'Connel et al. 63])

\section{Results of the Sobol' sensitivity analysis}

In this section (Tables A.1 to A.4, we provide original numerical values coming out from the sensitivity analysis. Values from Tables A.1 to A.4 were used to produce plots in Figure 6 and 7 in the main text, and Figure $\mathrm{A.2}$ and A.3. which were also used to discuss the results of the sensitivity analysis. 
Table A.1: First order and total Sobol sensitivity indices of the model outputs $n_{T G}$ for the hydrolysis at $180^{\circ} \mathrm{C}-1: 27.5$ oil-to-water molar ratio $-600 \mathrm{rpm}$

\begin{tabular}{c|c|c|c|c|c|c|c|c|c|c|c|c}
\hline \multicolumn{10}{c}{ First order Sobol' sensitivity indices } \\
\hline \multirow{3}{*}{$k_{1}$} & $\mathbf{0 . 5} \mathbf{~ h}$ & $\mathbf{1} \mathbf{~ h}$ & $\mathbf{1 . 5} \mathbf{h}$ & $\mathbf{2} \mathbf{~ h}$ & $\mathbf{2 . 5} \mathbf{h}$ & $\mathbf{3} \mathbf{h}$ & $\mathbf{3 . 5} \mathbf{h}$ & $\mathbf{4} \mathbf{h}$ & $\mathbf{4 . 5} \mathbf{h}$ & $\mathbf{5} \mathbf{~ h}$ & $\mathbf{5 . 5} \mathbf{~ h}$ & $\mathbf{6} \mathbf{~ h}$ \\
$k_{-1}$ & 0.522 & 0.584 & 0.583 & 0.483 & 0.379 & 0.300 & 0.241 & 0.193 & 0.155 & 0.126 & 0.102 & 0.084 \\
$k_{2}$ & 0.001 & 0.022 & 0.131 & 0.306 & 0.430 & 0.472 & 0.463 & 0.428 & 0.382 & 0.334 & 0.290 & 0.253 \\
$k_{-2}$ & 0.000 & 0.000 & 0.004 & 0.037 & 0.101 & 0.165 & 0.207 & 0.223 & 0.221 & 0.207 & 0.188 & 0.168 \\
$k_{3}$ & 0.000 & 0.000 & 0.000 & 0.002 & 0.013 & 0.041 & 0.084 & 0.130 & 0.169 & 0.195 & 0.208 & 0.212 \\
$k_{-3}$ & 0.000 & 0.000 & 0.000 & 0.002 & 0.002 & 0.001 & 0.000 & 0.003 & 0.009 & 0.018 & 0.026 & 0.031 \\
$k_{4}$ & 0.000 & 0.000 & 0.001 & 0.001 & 0.000 & 0.000 & 0.000 & 0.000 & 0.000 & 0.000 & 0.000 & 0.000 \\
$k_{-4}$ & 0.000 & 0.001 & 0.003 & 0.003 & 0.001 & 0.000 & 0.000 & 0.000 & 0.000 & 0.000 & 0.000 & 0.000 \\
$k_{1 L, a}$ & 0.477 & 0.392 & 0.278 & 0.166 & 0.071 & 0.017 & 0.001 & 0.017 & 0.054 & 0.104 & 0.160 & 0.215 \\
$k_{2 L, a}$ & 0.000 & 0.000 & 0.000 & 0.000 & 0.000 & 0.000 & 0.000 & 0.000 & 0.000 & 0.001 & 0.003 & 0.005 \\
\hline$\sum S_{i}$ & 1.000 & 1.000 & 1.000 & 0.999 & 0.998 & 0.997 & 0.996 & 0.994 & 0.993 & 0.990 & 0.987 & 0.985 \\
\hline \multicolumn{10}{|c|}{}
\end{tabular}

Total Sobol' sensitivity indices

\begin{tabular}{c|c|c|c|c|c|c|c|cc|c|c|c|c}
\hline & $\mathbf{0 . 5} \mathbf{h}$ & $\mathbf{1} \mathbf{h}$ & $\mathbf{1 . 5} \mathbf{h}$ & $\mathbf{2} \mathbf{h}$ & $\mathbf{2 . 5} \mathbf{h}$ & $\mathbf{3} \mathbf{h}$ & $\mathbf{3 . 5} \mathbf{h}$ & $\mathbf{4} \mathbf{h}$ & $\mathbf{4 . 5} \mathbf{h}$ & $\mathbf{5} \mathbf{h}$ & $\mathbf{5 . 5} \mathbf{h}$ & $\mathbf{6} \mathbf{h}$ \\
$k_{1}$ & 0.523 & 0.584 & 0.583 & 0.483 & 0.380 & 0.301 & 0.242 & 0.195 & 0.158 & 0.129 & 0.105 & 0.086 \\
$k_{-1}$ & 0.001 & 0.022 & 0.131 & 0.307 & 0.432 & 0.475 & 0.466 & 0.433 & 0.387 & 0.341 & 0.297 & 0.260 \\
$k_{2}$ & 0.000 & 0.000 & 0.005 & 0.038 & 0.102 & 0.167 & 0.209 & 0.226 & 0.224 & 0.211 & 0.192 & 0.172 \\
$k_{-2}$ & 0.000 & 0.000 & 0.000 & 0.002 & 0.013 & 0.042 & 0.084 & 0.131 & 0.171 & 0.198 & 0.213 & 0.218 \\
$k_{3}$ & 0.000 & 0.000 & 0.001 & 0.002 & 0.002 & 0.001 & 0.000 & 0.003 & 0.010 & 0.018 & 0.027 & 0.032 \\
$k_{-3}$ & 0.000 & 0.000 & 0.000 & 0.000 & 0.000 & 0.000 & 0.000 & 0.000 & 0.002 & 0.006 & 0.012 & 0.020 \\
$k_{4}$ & 0.000 & 0.000 & 0.001 & 0.001 & 0.000 & 0.000 & 0.000 & 0.000 & 0.000 & 0.000 & 0.000 & 0.000 \\
$k_{-4}$ & 0.000 & 0.001 & 0.003 & 0.003 & 0.001 & 0.000 & 0.000 & 0.000 & 0.000 & 0.000 & 0.000 & 0.000 \\
$k_{1 L, a}$ & 0.477 & 0.393 & 0.278 & 0.166 & 0.071 & 0.017 & 0.002 & 0.017 & 0.055 & 0.107 & 0.164 & 0.221 \\
$k_{2 L, a}$ & 0.000 & 0.000 & 0.000 & 0.000 & 0.000 & 0.000 & 0.000 & 0.000 & 0.000 & 0.001 & 0.003 & 0.006 \\
\hline$\sum S_{T i}$ & 1.000 & 1.000 & 1.001 & 1.001 & 1.002 & 1.003 & 1.004 & 1.006 & 1.007 & 1.010 & 1.013 & 1.015 \\
\hline
\end{tabular}


Table A.2: First order and total Sobol sensitivity indices of the model outputs $n_{D G}$ for the hydrolysis at $180^{\circ} \mathrm{C}-1: 27.5$ oil-to-water molar ratio $-600 \mathrm{rpm}$

\begin{tabular}{c|c|c|c|c|c|c|c|c|c|c|c|c}
\hline \multicolumn{10}{c}{ First order Sobol' sensitivity indices } \\
\hline \multirow{3}{*}{$k_{1}$} & $\mathbf{0 . 5} \mathbf{~ h}$ & $\mathbf{1} \mathbf{~ h}$ & $\mathbf{1 . 5} \mathbf{h}$ & $\mathbf{2} \mathbf{h}$ & $\mathbf{2 . 5} \mathbf{h}$ & $\mathbf{3} \mathbf{h}$ & $\mathbf{3 . 5} \mathbf{h}$ & $\mathbf{4} \mathbf{h}$ & $\mathbf{4 . 5} \mathbf{h}$ & $\mathbf{5} \mathbf{~ h}$ & $\mathbf{5 . 5} \mathbf{~ h}$ & $\mathbf{6} \mathbf{~ h}$ \\
$k_{-1}$ & 0.607 & 0.249 & 0.006 & 0.003 & 0.012 & 0.014 & 0.013 & 0.012 & 0.009 & 0.008 & 0.006 & 0.005 \\
$k_{2}$ & 0.001 & 0.020 & 0.022 & 0.015 & 0.006 & 0.002 & 0.000 & 0.000 & 0.000 & 0.001 & 0.000 & 0.000 \\
$k_{-2}$ & 0.000 & 0.708 & 0.788 & 0.749 & 0.714 & 0.657 & 0.584 & 0.509 & 0.439 & 0.376 & 0.325 & 0.274 \\
$k_{3}$ & 0.000 & 0.000 & 0.021 & 0.070 & 0.157 & 0.261 & 0.351 & 0.409 & 0.437 & 0.443 & 0.433 & 0.415 \\
$k_{-3}$ & 0.000 & 0.000 & 0.000 & 0.002 & 0.007 & 0.017 & 0.033 & 0.051 & 0.068 & 0.082 & 0.090 & 0.096 \\
$k_{4}$ & 0.000 & 0.001 & 0.001 & 0.001 & 0.000 & 0.000 & 0.000 & 0.000 & 0.000 & 0.000 & 0.000 & 0.000 \\
$k_{-4}$ & 0.001 & 0.008 & 0.005 & 0.003 & 0.001 & 0.001 & 0.000 & 0.000 & 0.000 & 0.000 & 0.000 & 0.000 \\
$k_{1 L, a}$ & 0.320 & 0.009 & 0.155 & 0.157 & 0.101 & 0.045 & 0.010 & 0.001 & 0.011 & 0.032 & 0.057 & 0.086 \\
$k_{2 L, a}$ & 0.000 & 0.000 & 0.000 & 0.000 & 0.000 & 0.000 & 0.001 & 0.002 & 0.005 & 0.011 & 0.020 & 0.031 \\
\hline$\sum S_{i}$ & 0.999 & 0.998 & 0.999 & 0.999 & 0.999 & 0.999 & 0.998 & 0.997 & 0.996 & 0.994 & 0.991 & 0.989 \\
\hline \multicolumn{10}{|c|}{}
\end{tabular}

Total Sobol' sensitivity indices

\begin{tabular}{c|c|c|c|c|c|c|c|cc|c|c|c|c}
\hline & $\mathbf{0 . 5} \mathbf{h}$ & $\mathbf{1} \mathbf{h}$ & $\mathbf{1 . 5} \mathbf{h}$ & $\mathbf{2} \mathbf{h}$ & $\mathbf{2 . 5} \mathbf{h}$ & $\mathbf{3} \mathbf{h}$ & $\mathbf{3 . 5} \mathbf{h}$ & $\mathbf{4} \mathbf{h}$ & $\mathbf{4 . 5} \mathbf{h}$ & $\mathbf{5} \mathbf{h}$ & $\mathbf{5 . 5} \mathbf{h}$ & $\mathbf{6} \mathbf{h}$ \\
$k_{1}$ & 0.608 & 0.250 & 0.007 & 0.004 & 0.012 & 0.014 & 0.013 & 0.012 & 0.009 & 0.008 & 0.006 & 0.005 \\
$k_{-1}$ & 0.001 & 0.020 & 0.022 & 0.015 & 0.006 & 0.002 & 0.000 & 0.000 & 0.001 & 0.001 & 0.001 & 0.001 \\
$k_{2}$ & 0.071 & 0.709 & 0.789 & 0.750 & 0.715 & 0.658 & 0.585 & 0.510 & 0.440 & 0.378 & 0.327 & 0.277 \\
$k_{-2}$ & 0.000 & 0.003 & 0.021 & 0.070 & 0.158 & 0.262 & 0.353 & 0.412 & 0.441 & 0.448 & 0.439 & 0.422 \\
$k_{3}$ & 0.000 & 0.000 & 0.001 & 0.002 & 0.007 & 0.018 & 0.034 & 0.052 & 0.070 & 0.084 & 0.093 & 0.099 \\
$k_{-3}$ & 0.000 & 0.000 & 0.000 & 0.000 & 0.000 & 0.002 & 0.006 & 0.014 & 0.027 & 0.044 & 0.062 & 0.086 \\
$k_{4}$ & 0.000 & 0.001 & 0.001 & 0.001 & 0.000 & 0.000 & 0.000 & 0.000 & 0.000 & 0.000 & 0.000 & 0.000 \\
$k_{-4}$ & 0.001 & 0.008 & 0.006 & 0.003 & 0.001 & 0.001 & 0.000 & 0.000 & 0.000 & 0.000 & 0.000 & 0.000 \\
$k_{1 L, a}$ & 0.321 & 0.010 & 0.156 & 0.157 & 0.101 & 0.046 & 0.010 & 0.001 & 0.011 & 0.032 & 0.059 & 0.088 \\
$k_{2 L, a}$ & 0.000 & 0.000 & 0.000 & 0.000 & 0.000 & 0.000 & 0.001 & 0.002 & 0.006 & 0.012 & 0.022 & 0.034 \\
\hline$\sum S_{T i}$ & 1.001 & 1.002 & 1.001 & 1.001 & 1.001 & 1.001 & 1.002 & 1.003 & 1.005 & 1.006 & 1.009 & 1.011 \\
\hline
\end{tabular}


Table A.3: First order and total Sobol sensitivity indices of the model outputs $n_{M G}$ for the hydrolysis at $180^{\circ} \mathrm{C}-1: 27.5$ oil-to-water molar ratio $-600 \mathrm{rpm}$

\begin{tabular}{c|c|c|c|c|c|c|c|c|c|c|c|c}
\hline \multicolumn{10}{c}{ First order Sobol' sensitivity indices } \\
\hline \multirow{3}{*}{$k_{1}$} & $\mathbf{0 . 5} \mathbf{~ h}$ & $\mathbf{1} \mathbf{~ h}$ & $\mathbf{1 . 5} \mathbf{~ h}$ & $\mathbf{2} \mathbf{h}$ & $\mathbf{2 . 5} \mathbf{h}$ & $\mathbf{3} \mathbf{h}$ & $\mathbf{3 . 5} \mathbf{h}$ & $\mathbf{4} \mathbf{h}$ & $\mathbf{4 . 5} \mathbf{h}$ & $\mathbf{5} \mathbf{~ h}$ & $\mathbf{5 . 5} \mathbf{~ h}$ & $\mathbf{6} \mathbf{~ h}$ \\
$k_{-1}$ & 0.138 & 0.142 & 0.110 & 0.034 & 0.003 & 0.000 & 0.002 & 0.003 & 0.003 & 0.003 & 0.003 & 0.003 \\
$k_{2}$ & 0.351 & 0.002 & 0.010 & 0.018 & 0.014 & 0.008 & 0.004 & 0.002 & 0.001 & 0.000 & 0.000 & 0.000 \\
$k_{-2}$ & 0.000 & 0.002 & 0.466 & 0.224 & 0.056 & 0.008 & 0.000 & 0.002 & 0.005 & 0.007 & 0.007 & 0.008 \\
$k_{3}$ & 0.003 & 0.049 & 0.290 & 0.047 & 0.052 & 0.044 & 0.032 & 0.022 & 0.013 & 0.009 & 0.005 & 0.003 \\
$k_{-3}$ & 0.000 & 0.000 & 0.003 & 0.020 & 0.060 & 0.122 & 0.196 & 0.281 & 0.345 & 0.386 & 0.410 & 0.415 \\
$k_{4}$ & 0.000 & 0.000 & 0.000 & 0.000 & 0.000 & 0.000 & 0.000 & 0.000 & 0.000 & 0.000 & 0.000 & 0.000 \\
$k_{-4}$ & 0.001 & 0.001 & 0.001 & 0.000 & 0.000 & 0.000 & 0.000 & 0.000 & 0.000 & 0.000 & 0.000 & 0.000 \\
$k_{1 L, a}$ & 0.504 & 0.349 & 0.098 & 0.002 & 0.030 & 0.038 & 0.022 & 0.007 & 0.001 & 0.003 & 0.009 & 0.016 \\
$k_{2 L, a}$ & 0.000 & 0.000 & 0.000 & 0.001 & 0.003 & 0.011 & 0.027 & 0.052 & 0.086 & 0.129 & 0.175 & 0.217 \\
\hline$\sum S_{i}$ & 0.997 & 0.999 & 0.998 & 0.997 & 0.999 & 0.998 & 0.996 & 0.993 & 0.990 & 0.986 & 0.982 & 0.978 \\
\hline \multicolumn{10}{|c|}{}
\end{tabular}

Total Sobol' sensitivity indices

\begin{tabular}{c|c|c|c|c|c|c|c|c|c|c|c|c}
\hline & $\mathbf{0 . 5} \mathbf{h}$ & $\mathbf{1} \mathbf{h}$ & $\mathbf{1 . 5} \mathbf{h}$ & $\mathbf{2} \mathbf{h}$ & $\mathbf{2 . 5} \mathbf{h}$ & $\mathbf{3} \mathbf{h}$ & $\mathbf{3 . 5} \mathbf{h}$ & $\mathbf{4} \mathbf{h}$ & $\mathbf{4 . 5} \mathbf{h}$ & $\mathbf{5} \mathbf{h}$ & $\mathbf{5 . 5} \mathbf{h}$ & $\mathbf{6} \mathbf{h}$ \\
$k_{1}$ & 0.139 & 0.142 & 0.111 & 0.035 & 0.004 & 0.000 & 0.002 & 0.003 & 0.004 & 0.003 & 0.003 & 0.003 \\
$k_{-1}$ & 0.000 & 0.002 & 0.010 & 0.018 & 0.014 & 0.008 & 0.005 & 0.002 & 0.001 & 0.000 & 0.000 & 0.000 \\
$k_{2}$ & 0.354 & 0.455 & 0.467 & 0.225 & 0.057 & 0.008 & 0.001 & 0.003 & 0.006 & 0.008 & 0.008 & 0.008 \\
$k_{-2}$ & 0.000 & 0.002 & 0.020 & 0.048 & 0.052 & 0.044 & 0.033 & 0.023 & 0.015 & 0.010 & 0.006 & 0.004 \\
$k_{3}$ & 0.003 & 0.049 & 0.292 & 0.654 & 0.780 & 0.768 & 0.713 & 0.626 & 0.535 & 0.450 & 0.375 & 0.318 \\
$k_{-3}$ & 0.000 & 0.000 & 0.003 & 0.020 & 0.061 & 0.123 & 0.199 & 0.286 & 0.355 & 0.399 & 0.426 & 0.436 \\
$k_{4}$ & 0.000 & 0.000 & 0.000 & 0.000 & 0.000 & 0.000 & 0.000 & 0.000 & 0.000 & 0.000 & 0.000 & 0.000 \\
$k_{-4}$ & 0.001 & 0.001 & 0.001 & 0.000 & 0.000 & 0.000 & 0.000 & 0.000 & 0.000 & 0.000 & 0.000 & 0.000 \\
$k_{1 L, a}$ & 0.507 & 0.349 & 0.099 & 0.003 & 0.031 & 0.038 & 0.023 & 0.007 & 0.001 & 0.003 & 0.009 & 0.017 \\
$k_{2 L, a}$ & 0.000 & 0.000 & 0.000 & 0.001 & 0.004 & 0.012 & 0.030 & 0.058 & 0.095 & 0.141 & 0.191 & 0.237 \\
\hline$\sum S_{T i}$ & 1.003 & 1.001 & 1.002 & 1.003 & 1.001 & 1.002 & 1.004 & 1.007 & 1.010 & 1.014 & 1.018 & 1.022 \\
\hline
\end{tabular}


Table A.4: First order and total Sobol sensitivity indices of the model outputs $n_{F A}$ for the hydrolysis at $180^{\circ} \mathrm{C}-1: 27.5$ oil-to-water molar ratio $-600 \mathrm{rpm}$

\begin{tabular}{c|c|c|c|c|c|c|c|c|c|c|c|c}
\hline \multicolumn{10}{c}{ First order Sobol' sensivitity indices } \\
\hline \multirow{3}{*}{$k_{1}$} & $\mathbf{0 . 5} \mathbf{~ h}$ & $\mathbf{1} \mathbf{~ h}$ & $\mathbf{1 . 5} \mathbf{~ h}$ & $\mathbf{2} \mathbf{h}$ & $\mathbf{2 . 5} \mathbf{h}$ & $\mathbf{3} \mathbf{h}$ & $\mathbf{3 . 5} \mathbf{h}$ & $\mathbf{4} \mathbf{h}$ & $\mathbf{4 . 5} \mathbf{h}$ & $\mathbf{5} \mathbf{~ h}$ & $\mathbf{5 . 5} \mathbf{~ h}$ & $\mathbf{6} \mathbf{~ h}$ \\
$k_{-1}$ & 0.421 & 0.318 & 0.229 & 0.171 & 0.134 & 0.107 & 0.084 & 0.066 & 0.052 & 0.040 & 0.032 & 0.026 \\
$k_{2}$ & 0.001 & 0.008 & 0.024 & 0.042 & 0.055 & 0.062 & 0.064 & 0.062 & 0.056 & 0.051 & 0.044 & 0.039 \\
$k_{-2}$ & 0.142 & 0.279 & 0.376 & 0.421 & 0.423 & 0.392 & 0.341 & 0.291 & 0.240 & 0.201 & 0.167 \\
$k_{3}$ & 0.000 & 0.001 & 0.006 & 0.024 & 0.058 & 0.102 & 0.144 & 0.172 & 0.184 & 0.189 & 0.182 & 0.174 \\
$k_{-3}$ & 0.000 & 0.006 & 0.032 & 0.082 & 0.143 & 0.198 & 0.234 & 0.245 & 0.240 & 0.223 & 0.204 & 0.185 \\
$k_{4}$ & 0.000 & 0.000 & 0.000 & 0.002 & 0.010 & 0.028 & 0.058 & 0.093 & 0.126 & 0.153 & 0.177 & 0.192 \\
$k_{-4}$ & 0.000 & 0.000 & 0.000 & 0.000 & 0.000 & 0.000 & 0.000 & 0.000 & 0.000 & 0.000 & 0.000 & 0.000 \\
$k_{1 L, a}$ & 0.551 & 0.526 & 0.429 & 0.303 & 0.178 & 0.075 & 0.016 & 0.002 & 0.017 & 0.052 & 0.086 & 0.121 \\
$k_{2 L, a}$ & 0.000 & 0.000 & 0.000 & 0.000 & 0.001 & 0.003 & 0.007 & 0.016 & 0.030 & 0.047 & 0.066 & 0.085 \\
\hline$\sum S_{i}$ & 0.999 & 0.999 & 1.000 & 1.000 & 0.999 & 0.999 & 0.999 & 0.998 & 0.996 & 0.995 & 0.992 & 0.989 \\
\hline \multicolumn{10}{|c|}{} \\
\hline
\end{tabular}

Total Sobol' sensitivity indices

\begin{tabular}{c|c|c|c|c|c|c|c|cc|c|c|c|c}
\hline & $\mathbf{0 . 5} \mathbf{h}$ & $\mathbf{1} \mathbf{h}$ & $\mathbf{1 . 5} \mathbf{h}$ & $\mathbf{2} \mathbf{h}$ & $\mathbf{2 . 5} \mathbf{h}$ & $\mathbf{3} \mathbf{h}$ & $\mathbf{3 . 5} \mathbf{h}$ & $\mathbf{4} \mathbf{h}$ & $\mathbf{4 . 5} \mathbf{h}$ & $\mathbf{5} \mathbf{h}$ & $\mathbf{5 . 5} \mathbf{h}$ & $\mathbf{6} \mathbf{h}$ \\
$k_{1}$ & 0.422 & 0.318 & 0.229 & 0.171 & 0.134 & 0.107 & 0.084 & 0.066 & 0.052 & 0.040 & 0.032 & 0.026 \\
$k_{-1}$ & 0.001 & 0.008 & 0.024 & 0.042 & 0.055 & 0.063 & 0.065 & 0.062 & 0.056 & 0.051 & 0.045 & 0.040 \\
$k_{2}$ & 0.027 & 0.143 & 0.280 & 0.376 & 0.421 & 0.424 & 0.392 & 0.341 & 0.291 & 0.240 & 0.201 & 0.168 \\
$k_{-2}$ & 0.000 & 0.001 & 0.006 & 0.024 & 0.059 & 0.103 & 0.144 & 0.173 & 0.185 & 0.190 & 0.183 & 0.176 \\
$k_{3}$ & 0.000 & 0.006 & 0.032 & 0.082 & 0.143 & 0.198 & 0.234 & 0.245 & 0.241 & 0.224 & 0.205 & 0.185 \\
$k_{-3}$ & 0.000 & 0.000 & 0.000 & 0.002 & 0.010 & 0.029 & 0.059 & 0.095 & 0.129 & 0.157 & 0.184 & 0.200 \\
$k_{4}$ & 0.000 & 0.000 & 0.000 & 0.000 & 0.000 & 0.000 & 0.000 & 0.000 & 0.000 & 0.000 & 0.000 & 0.000 \\
$k_{-4}$ & 0.000 & 0.000 & 0.000 & 0.000 & 0.000 & 0.000 & 0.000 & 0.000 & 0.000 & 0.000 & 0.000 & 0.000 \\
$k_{1 L, a}$ & 0.551 & 0.526 & 0.429 & 0.303 & 0.178 & 0.076 & 0.016 & 0.003 & 0.018 & 0.052 & 0.087 & 0.123 \\
$k_{2 L, a}$ & 0.000 & 0.000 & 0.000 & 0.000 & 0.001 & 0.003 & 0.008 & 0.018 & 0.032 & 0.051 & 0.072 & 0.093 \\
\hline$\sum S_{T i}$ & 1.001 & 1.001 & 1.000 & 1.000 & 1.001 & 1.001 & 1.001 & 1.002 & 1.004 & 1.005 & 1.008 & 1.011 \\
\hline
\end{tabular}




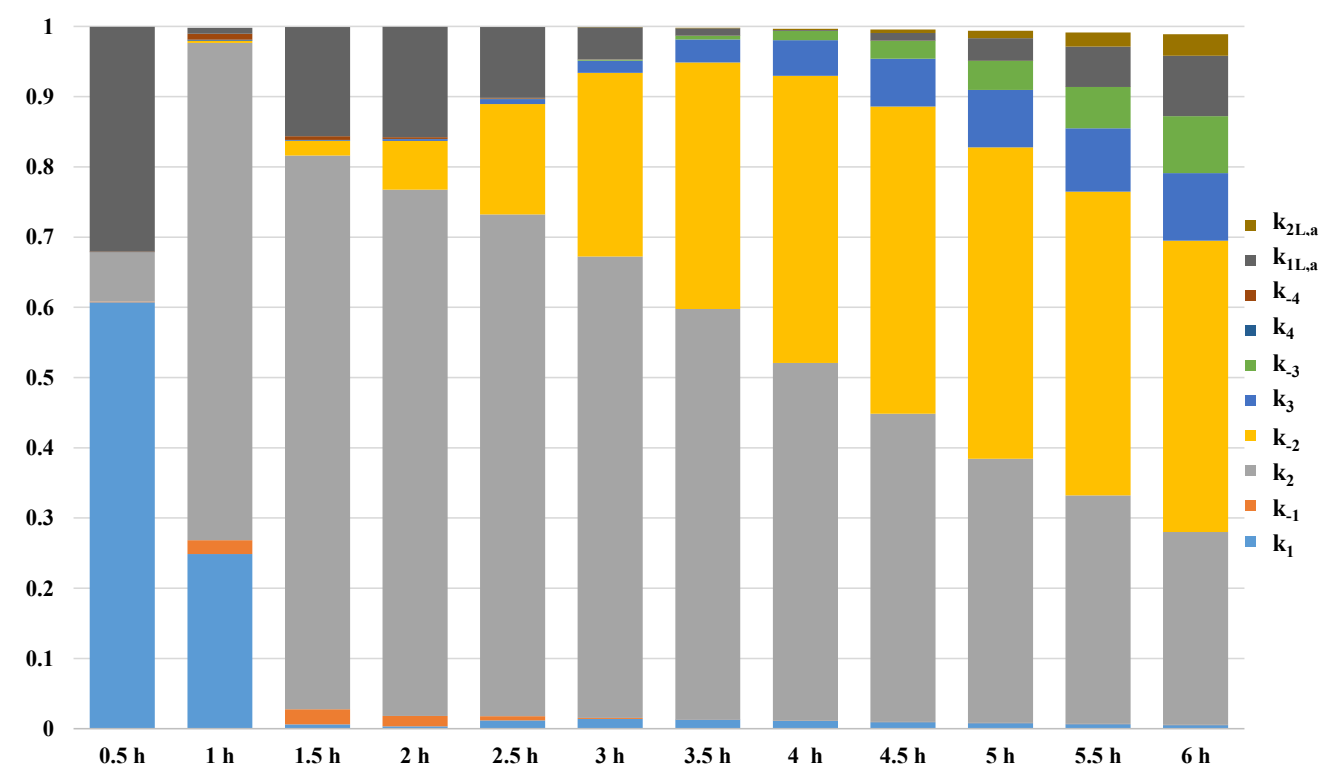

Figure A.2: Contribution to the variations in the model output $n_{D G}$ over time using Sobol sensitivity indices for the hydrolysis at $180^{\circ} \mathrm{C}-1: 27.5$ oil-to-water molar ratio - $600 \mathrm{rpm}$

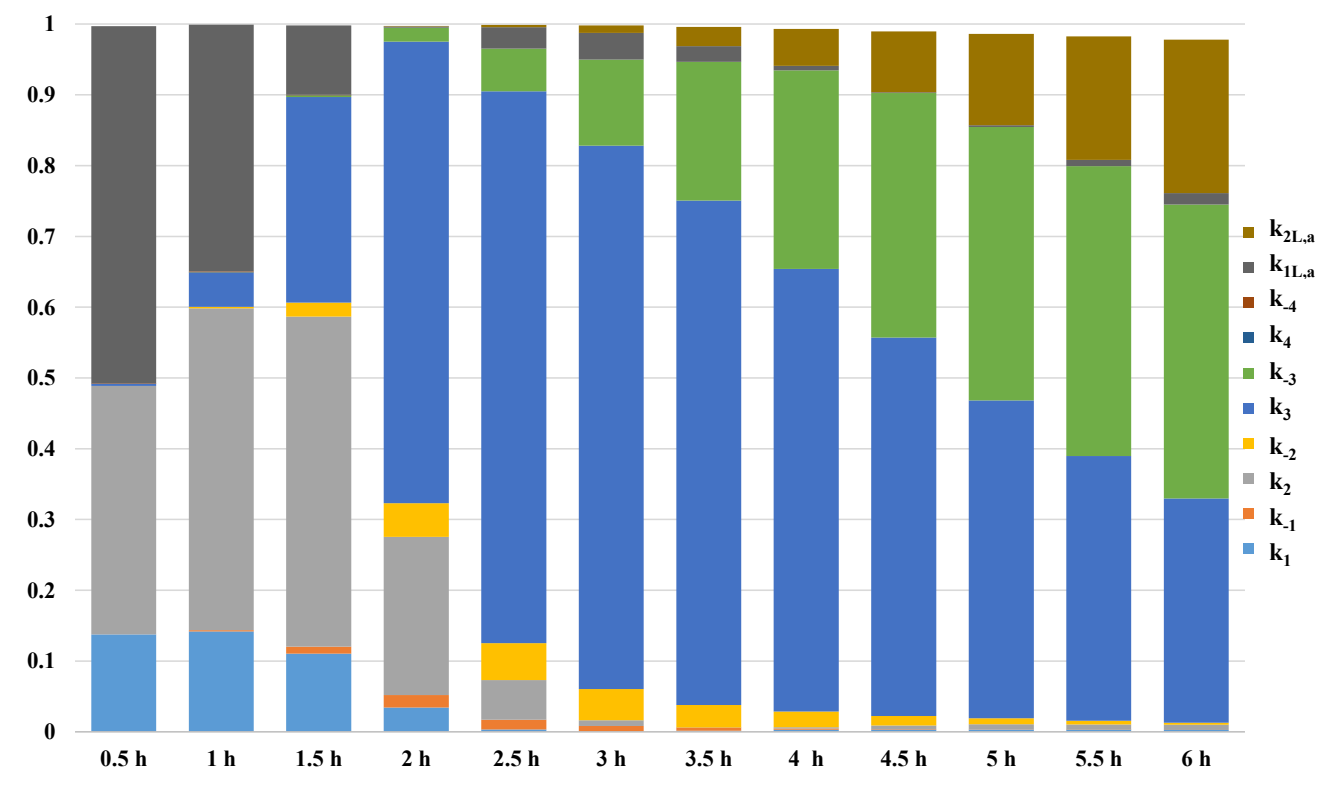

Figure A.3: Contribution to the variations in the model output $n_{M G}$ over time using Sobol sensitivity indices for the hydrolysis at $180^{\circ} \mathrm{C}-1: 27.5$ oil-to-water molar ratio - $600 \mathrm{rpm}$ 